\title{
FOURIER METHOD FOR FIRST ORDER DIFFERENTIAL EQUATIONS WITH INVOLUTION AND GROUPS OF OPERATORS
}

\author{
A.G. BASKAKOV, N.B. USKOVA
}

\begin{abstract}
In the paper we study a mixed problem for a first-order differential equation with an involution. It is written by means of a differential operator with an involution acting in the space functions square integrable on a finite interval. We construct a similarity transform of this operator in an operator being an orthogonal direct sum of an operator of finite rank and operators of rank 1. The method of our study is the method of similar operators. Theorem on similarity serves as the basis for constructing groups of operators, whose generator is the original operator. We write out asymptotic formulae for groups of operators. The constructed group allows us to introduce the notion of a mild solution, and also to describe the mild solutions to the considered problem.

This serves to justify the Fourier method. Almost periodicity of bounded mild solutions is established. The proof of almost periodicity is based on the asymptotic representation of the spectrum of a differential operator with an involution.
\end{abstract}

Keywords: method of similar operator, spectrum, mixed problem, group of operators, differential operator with involution.

Mathematics Subject Classification: 34L15, 34B09, 47E05

\section{INTRODUCTION AND MAIN RESULTS}

We study the mixed problem for a hyperbolic equation with an involution:

$$
\left\{\begin{array}{l}
\frac{\partial u(t, s)}{\partial t}=\frac{\partial u(t, s)}{\partial s}-v(s) u(t, \omega-s) \\
u(t, 0)=u(t, \omega) \\
u(0, s)=\varphi(s) \\
t \in \mathcal{J}, \quad s \in[0, \omega]
\end{array}\right.
$$

where the symbol $\mathcal{J}$ denotes of the intervals $(-\infty, \infty),(-\infty, \beta],[\alpha, \beta],[\alpha, \infty)$. Hereinafter we assume that the considered interval contains zero.

A.G. BASKAKOV, N.B. UsKova, FOURIER METHOD FOR FIRST ORDER DIFFERENTIAL EQUATIONS With INVOLUTION AND GROUPS OF OPERATORS.

(c) Baskakov A.G., Uskova N.B. 2018.

The work of the first author is supported by the Ministry of Equcation and Science of Russia in the framework of the project part of state task (project no. 1.3464.2017/4.6). The work of the second author is supported by RFBR (project no. 16-01-00197).

Submitted June 29, $201 \%$. 

lem

Together with homogeneous problem (1) we consider the corresponding inhomogeneous prob-

$$
\left\{\begin{array}{l}
\frac{\partial u(t, s)}{\partial t}=\frac{\partial u(t, s)}{\partial s}-v(s) u(t, \omega-s)+f(t, s) \\
u(t, 0)=u(t, \omega) \\
u(0, s)=\varphi(s) \\
t \in \mathcal{J}, \quad s \in[0, \omega] .
\end{array}\right.
$$

To formulate the problem, we introduce the following functional spaces. By $L_{2}=L_{2}[0, \omega]$ we denote the Hilbert space of Lebesgue measurable square integrable functions (equivalence classes) on $[0, \omega]$ with values in $\mathbb{C}$. The scalar product in $L_{2}$ is defined by the formula

$$
(x, y)=\frac{1}{\omega} \int_{0}^{\omega} x(s) \overline{y(s)} d s, \quad x, y \in L_{2},
$$

and the norm is generated by this scalar product.

The space $L_{2}$ is isometrically isomorphic to the Hilbert space $L_{2, \omega}=L_{2, \omega}(\mathbb{R}, \mathbb{C})$ of complex $\omega$-periodic functions defined on the entire axis $\mathbb{R}$ and square integrable on $[0, \omega]$. In what follows, each function $x \in L_{2}$ will be identified with its $\omega$-periodic continuation on $\mathbb{R}$.

By $W_{2}^{1}=W_{2}^{1}[0, \omega]$ we denote the Sobolev space of absolutely continuous functions in $L_{2}$ with derivatives in $L_{2}$; this space is equipped with the scalar product $(x, y)_{W_{2}^{1}}=(x, y)+\left(x^{\prime}, y^{\prime}\right), x$, $y \in W_{2}^{1}$.

By symbol End $\mathcal{H}$ we denote the Banach algebra of bounded linear operators acting in an abstract Hilbert space $\mathcal{H}$ with the norm

$$
\|X\|_{\infty}=\sup _{\|x\| \leqslant 1}\|X x\|, \quad x \in \mathcal{H}, \quad X \in \operatorname{End} \mathcal{H} .
$$

We introduce a two-sided ideal of Hilbert-Schmidt operators $\mathfrak{S}_{2}(\mathcal{H})$ in the algebra End $\mathcal{H}$. By $\|X\|_{2}$ we denote the norm of Hilbert-Schmidt operators $X \in \mathfrak{S}_{2}(\mathcal{H})$, that is, $\|X\|_{2}=$ $\left(\operatorname{tr}\left(X X^{*}\right)\right)^{1 / 2}$. Here $\operatorname{tr}\left(X X^{*}\right)$ is the trace of the operator $X X^{*}$ belonging to a two-sided ideal $\mathfrak{S}_{1}(\mathcal{H})$ of nuclear operators in End $\mathcal{H}$ (see [1]) with the norm $\|X\|_{1}=\operatorname{tr}\left(X X^{*}\right)=\sum_{n \in \mathbb{Z}} s_{n}$, where $\left(s_{n}\right)$ is the sequence of $s$-numbers of an operator $X$. The formula $(X, Y)=\operatorname{tr}\left(X Y^{*}\right), X$, $Y \in \mathfrak{S}_{2}(\mathcal{H})$, defines a scalar product in $\mathfrak{S}_{2}(\mathcal{H})$.

In the paper we assume that the potential $v$ belongs to the space $L_{2}$. In what follows, by the symbol $C\left(\mathcal{J}, L_{2}\right)$ we denote a linear space of functions $u: \mathcal{J} \times[0, \omega] \rightarrow \mathbb{C}$ possessing the following properties. For a fixed $t \in \mathcal{J}$, the function $s \mapsto u(t, s)$ belongs $L_{2}$. Moreover, the function

$$
\widetilde{u}: \mathcal{J} \rightarrow L_{2}, \quad \widetilde{u}(t)(s)=u(t, s), \quad t \in \mathcal{J}, \quad s \in[0, \omega]
$$

is assumed to be continuous. If $\mathcal{J}$ is a finite interval, then the space $C\left(\mathcal{J}, L_{2}\right)$ is Banach. In this case as the norm, the quantity $\|u\|_{\infty}=\max _{t \in \mathcal{J}}\|\widetilde{u}(t)\|_{2}$ serves. The function $\widetilde{u}$ is called associated with the function $u$ and they will be identified.

The function $f: \mathcal{J} \times[0, \omega] \rightarrow \mathbb{C}$ in inhomogeneous problem (2) is assumed to belong to the space $C\left(\mathcal{J}, L_{2}\right)$.

We mention a series of works [2]-[5], where mixed problem (1) was considered with a smooth potential $v:[0, \omega] \rightarrow \mathbb{C}$. In these works, the problem on justification of Fourier method for homogeneous problem (1) was studied by a resolvent method (by means of contour integration). There was also studied the asymptotics of eigenvalues and equiconvergence of spectral resolutions for the $L$ defined by problem (1). We shall define this operator a little bit later. 
Second order differential operators with involution were studied in papers [6]-[8]. In work [8] there was given a complete bibliographic survey of results on equiconvergence of spectral resolutions for first and second order operators with involution.

A qualitative analysis of solutions to boundary value problems for first and second order equations with involutions was made in [9]-[13]. The basicity property of root functions were considered in [14]. The operator generated by a second order differential expression with an involution at a derivative was studied in [15, [16].

The spectral properties of the operator $L$ (the asymptotics of eigenvalues and estimates for the rate of equiconvergence of spectral resolutions) for a system of differential equations with involution were obtained by the method of similar operators in [17]. In the present work, the main approach is also the method of similar operators. We also mention papers [18]-[22], in which the method of similar operators was developed.

Operators with involution arise in the filtration theory, theory of prediction and in studying subharmonic oscillations [23]-[26].

Problems (1) and (2) in Hilbert space $L_{2}$ are written respectively as

$$
\begin{aligned}
& \widetilde{u}_{t}^{\prime}=L \widetilde{u}, \quad \widetilde{u}(0)=\varphi, \\
& \widetilde{u}_{t}^{\prime}=L \widetilde{u}+\widetilde{f}, \quad \widetilde{u}(0)=\varphi .
\end{aligned}
$$

The operator $L: D(L) \subset L_{2} \rightarrow L_{2}$ in equations (3), (4) is defined by the formula

$$
(L y)(s)=\frac{d y(s)}{d s}-v(s) y(\omega-s), \quad s \in[0, \omega] .
$$

Its domain $D(L)$ is defined by periodic boundary conditions

$$
D(L)=\left\{y \in W_{2}^{1}: y(0)=y(\omega)\right\} .
$$

Let us formulate definitions related to the notion of a solution to considered Cauchy problems (1), (2).

Definition 1. ([27]) A classical solution to problem (2) is a continuously differentiable function $u: \mathcal{J} \times[0, \omega] \rightarrow \mathbb{C}$ belonging to the space $C\left(\mathcal{J}, L_{2}\right)$ such that the associated function $\widetilde{u}: \mathcal{J} \rightarrow L_{2}$ is continuously differentiable and for each $t \in \mathcal{J}$ it satisfies the condition $\widetilde{u}(t) \in D(L)$ and equation (4).

Definition 2. ([27, § 3.1]) A function $u \in C\left(\mathcal{J}, L_{2}\right)$ is called a mild solution to problem (4) if $\int_{0}^{t} \widetilde{u}(s) d s \in D(L)$ for each $t \in \mathcal{J}$ and

$$
\widetilde{u}(t)=\varphi+L \int_{0}^{t} \widetilde{u}(s) d s+\int_{0}^{t} \widetilde{f}(s) d s, \quad t \in \mathcal{J},
$$

where the Riemann integrals are considered for continuous functions defined on $\mathcal{J}$ with values in Hilbert space $L_{2}$.

The next definition follows naturally Definition 1 .

Definition 3. A classical solution to problem (1), where $\varphi \in W_{2}^{1}$, is a function $u: \mathcal{J} \times$ $[0, \omega] \rightarrow \mathbb{C}$ belonging to the space $C\left(\mathcal{J}, L_{2}\right)$ such that the associated function $\widetilde{u}: \mathcal{J} \rightarrow L_{2}$ is continuously differentiable and solves problem (1).

Definition 4. A function $\widetilde{u}: \mathcal{J} \rightarrow L_{2}$ is called a mild solution to problem (1) if it is a uniform limit on each bounded interval in $\mathcal{J}$ of classical solutions $\left(\widetilde{u}_{n}\right), n \geqslant 1$, obeying $\widetilde{u}_{n}(0)=\varphi_{n} \in W_{2}^{1}, n \geqslant 1$, where $\lim _{n \rightarrow \infty} \varphi_{n}=\varphi$.

Definition 5. Problem (1) is said to be uniformly well-defined if for each initial condition $\varphi \in L_{2}$ there exists a unique mild solution $x \in C\left(\mathcal{J}, L_{2}\right)$ obeying the condition $\widetilde{x}(0)=\varphi$. 
A close definition of a well-defined problem for an abstract differential operator with a constant operator coefficient was given in monograph [28, Ch. II, Sect. 3]. We observe that the definition of a well-definite problem (1) is equivalent to the fact the operator $L$ is a generator of a strongly continuous group of operators.

In this paper, using the theory of semi-groups of operators will play a key role in justification of Fourier method for the equation with involution.

Theorem 1. Problem (1) is uniformly well-defined. The differential operator L is a generator of some strongly continuous group of operators

$$
T: \mathbb{R} \rightarrow \text { End } L_{2} \text {. }
$$

Each classical solution $u \in C\left(\mathcal{J}, L_{2}\right)$ to problem (1) is defined by the formula

$$
u(t, s)=(T(t) \varphi)(s), \quad s \in[0, \omega], \quad t \in \mathcal{J},
$$

where $\varphi \in W_{2}^{1}$ and $\varphi(0)=\varphi(\omega)$. Each mild solution is given by (7), where $\varphi \in L_{2}$.

Owing to application of the method of similar operators, further Theorem 1 will be significantly strengthened, see Theorems 6 8. It should be noted that at least for a bounded function $v$, Theorem 1 can be obtained on the base of general theorems on perturbed semi-groups (groups) of operators (see [29], [30]).

Remark 1. It follows from [27, Prop 3.1.16] that each mild solution $u \in C\left(\mathcal{J}, L_{2}\right)$ to problem (2) written as (4) can be represented as

$$
\widetilde{u}(t)=T\left(t-t_{0}\right) \widetilde{u}\left(t_{0}\right)-\int_{t_{0}}^{t} T(t-\tau) \widetilde{f}(\tau) d \tau, \quad t, t_{0} \in \mathcal{J}
$$

where $T: \mathbb{R} \rightarrow$ End $L_{2}$ is the group of operators in Theorem 1 , whose generator is the operator $L$.

In view of Remark 1, the following definition of classical solution is equivalent to Definition 1 .

Definition 6. A classical solution to problem (2) is a function $u \in C\left(\mathcal{J}, L_{2}\right)$ satisfying identity (8) (integral equation) such that $\widetilde{u}(t) \in D(L) \subset W_{2}^{1}$.

Thus, classical solution satisfies identity (4).

Theorem 2. Cauchy problem (2) has a unique mild solution $x \in C\left(\mathcal{J}, L_{2}\right)$ such that the associated function $\widetilde{x}$ can be represented as

$$
\widetilde{x}(t)=T(t) \varphi+\int_{0}^{t} T(t-\tau) \widetilde{f}(\tau) d \tau, \quad t \in \mathcal{J}
$$

The statement of Theorem 2 follows Theorem 1 and Definition 6 .

Throughout the rest of the paper are regularly employed the following definition and properties of similar operators.

Definition 7. Two linear operators $A_{i}: D\left(A_{i}\right) \subset \mathcal{H} \rightarrow \mathcal{H}, i=1,2$, are called similar if there exists a continuously invertible operator $U \in$ End $\mathcal{H}$ such that

$$
A_{1} U x=U A_{2} x, \quad x \in D\left(A_{2}\right), \quad U D\left(A_{2}\right)=D\left(A_{1}\right) .
$$

The operator $U$ is called the operator of transformation operator $A_{1}$ into $A_{2}$.

Such operators possess a series of coinciding spectral properties. It is convenient to formulate an appropriate statement as the following lemma. 
Lemma 1. Let $A_{i}: D\left(A_{i}\right) \subset \mathcal{H} \rightarrow \mathcal{H}, i=1,2$, be two similar operators and $U \in$ End $\mathcal{H}$ is the operator of transformation operator $A_{1}$ into operator $A_{2}$. Then the following statements are true:

1) $\sigma\left(A_{1}\right)=\sigma\left(A_{2}\right), \sigma_{d}\left(A_{1}\right)=\sigma_{d}\left(A_{2}\right), \sigma_{c}\left(A_{1}\right)=\sigma_{c}\left(A_{2}\right), \sigma_{r}\left(A_{1}\right)=\sigma_{r}\left(A_{2}\right)$, where $\sigma\left(A_{i}\right), \sigma_{d}\left(A_{i}\right)$, $\sigma_{c}\left(A_{i}\right), \sigma_{r}\left(A_{i}\right), i=1,2$, are the spectrum, the discrete spectrum. the continuous spectrum and the residual spectrum of the operators $A_{i}, i=1,2$, respectively;

2) if the operator $A_{2}$ admits the expansion $A_{2}=A_{21} \oplus A_{22}$, where $A_{2 k}=A_{2} \mid \mathcal{H}_{k}, k=1,2$, is the restriction of $A_{2}$ on $\mathcal{H}_{k}$ with respect to the direct sum $\mathcal{H}=\mathcal{H}_{1} \oplus \mathcal{H}_{2}$ of subspaces $\mathcal{H}_{1}, \mathcal{H}_{2}$ invariant with respect to $A_{2}$, then subspaces $\widetilde{\mathcal{H}}_{k}=U\left(\mathcal{H}_{k}\right), k=1,2$, are invariant with respect to the operator $A_{1}$ and $A_{1}=A_{11} \oplus A_{12}$, where $A_{1 k}=A_{1} \mid \widetilde{\mathcal{H}}_{k}, k=1,2$, at that, $\mathcal{H}=\widetilde{\mathcal{H}}_{1} \oplus \widetilde{\mathcal{H}}_{2}$. Moreover, if $P$ is a projector corresponding to the expansion $\mathcal{H}=\mathcal{H}_{1} \oplus \mathcal{H}_{2}$, that is, $\mathcal{H}_{1}=\operatorname{Im} P$ is the image of the projector $P, \mathcal{H}_{2}=\operatorname{Im}(I-P)$ is the image of the additional projector $I-P$, then the projector $\widetilde{P} \in$ End $\mathcal{H}$ corresponding to the expansion $\mathcal{H}=\widetilde{\mathcal{H}}_{1} \oplus \widetilde{\mathcal{H}}_{2}$ is defined by the formula

$$
\widetilde{P}=U P U^{-1} \text {. }
$$

3) if $a$ is an eigenvector of the operator $A_{2}$ associated with the eigenvalue $\lambda_{0}$, then $b=U a$ is an eigenvector of the operator $A_{1}$ associated with the same eigenvalue $\lambda_{0}$.

4) if the operator $A_{2}$ is a generator of a strongly continuous semi-group (group) of the operators $T_{2}: \mathbb{J} \rightarrow$ End $\mathcal{H}$ (of class $C_{0}$ ), then the operator $A_{1}$ is a generator a strongly continuous semi-group (group) of operators

$$
T_{1}(t)=U T_{2}(t) U^{-1}, \quad t \in \mathbb{J}, \quad T_{1}: \mathbb{J} \rightarrow \operatorname{End} \mathcal{H},
$$

where $\mathbb{J}$ coincides with one of the sets $\mathbb{R}_{+}, \mathbb{R}$.

Let $\mathcal{H}$ be an abstract Hilbert space represented as the direct sum of orthogonal non-zero closed subspaces $\mathcal{H}_{n}, n \in \mathbb{Z}$, that is,

$$
\mathcal{H}=\bigoplus_{n \in \mathbb{Z}} \mathcal{H}_{n}
$$

where $\mathcal{H}_{i}$ is orthogonal to $\mathcal{H}_{j}$ as $i \neq j, i, j \in \mathbb{Z}$, and $x=\sum_{n \in \mathbb{Z}} x_{n}, x_{n} \in \mathcal{H}_{n},\|x\|^{2}=\sum_{n \in \mathbb{Z}}\left\|x_{n}\right\|^{2}$. Such representation of the space $\mathcal{H}$ gives rise the partition of the unity of the system of orthoprojectors $\left\{\mathcal{P}_{n}, n \in \mathbb{Z}\right\}$. The projectors $\mathcal{P}_{n}, n \in \mathbb{Z}$, possess the following properties:

1) $\mathcal{P}_{n}^{*}=\mathcal{P}_{n}, n \in \mathbb{Z}$

2) $\mathcal{P}_{i} \mathcal{P}_{j}=0$ as $i \neq j, i, j \in \mathbb{Z}$

3) the series $\sum_{n \in \mathbb{Z}} \mathcal{P}_{n} x$ unconditionally converges to $x \in \mathcal{H}$ and $\|x\|^{2}=\sum_{n \in \mathbb{Z}}\left\|\mathcal{P}_{n} x\right\|^{2}$;

3') the identities $\mathcal{P}_{k} x=0, k \in \mathbb{Z}$ imply that the vector $x$ is zero;

4) $\mathcal{H}_{k}=\operatorname{Im} \mathcal{P}_{k}, x_{k}=\mathcal{P}_{k} x, k \in \mathbb{Z}$

We observe that the properties 3) and 3') are equivalent.

Definition 8. A linear operator $\mathcal{A}: D(\mathcal{A}) \subset \mathcal{H} \rightarrow \mathcal{H}$ is called an orthogonal direct sum of bounded operators $\mathcal{A}_{n} \in$ End $\mathcal{H}_{n}, n \in \mathbb{Z}$, with respect to resolution (9) and it is written as

$$
\mathcal{A}=\bigoplus_{n \in \mathbb{Z}} \mathcal{A}_{n}
$$

if

1) $\mathcal{H}_{n} \subset D(\mathcal{A})=\left\{x \in \mathcal{H}: \sum_{k \in \mathbb{Z}}\left\|\mathcal{A}_{k} x_{k}\right\|^{2}<\infty, x_{k}=\mathcal{P}_{k} x, k \in \mathbb{Z}\right\}$ for all $n \in \mathbb{Z}$;

2) each subspace $\mathcal{H}_{n}, n \in \mathbb{Z}$, is invariant with respect to the operator $\mathcal{A}$ and $\mathcal{A}_{n}, n \in \mathbb{Z}$, is the restriction of the operator $\mathcal{A}$ on $\mathcal{H}_{n}, n \in \mathbb{Z}$.

3) $\mathcal{A} x=\sum_{k \in \mathbb{Z}} \mathcal{A}_{k} x_{k}, x \in D(\mathcal{A})$, where $x_{k}=\mathcal{P}_{k} x, k \in \mathbb{Z}$. 
We observe the inclusion of the spectra: $\sigma\left(\mathcal{A}_{k}\right) \subset \sigma(\mathcal{A}), k \in \mathbb{Z}$.

Without additional restrictions, the spectrum $\sigma(\mathcal{A})$ does not necessarily coincide with the union of the spectra $\sigma\left(\mathcal{A}_{k}\right), k \in \mathbb{Z}$, and even with the closure of the union.

Definition 9. The expansion of the Hilbert space $\mathcal{H}$ of form

$$
\mathcal{H}=\bigoplus_{k \in \mathbb{Z}} U \mathcal{H}_{k}
$$

where $U$ is the invertible operator in End $\mathcal{H}$ and $\mathcal{H}$ is the orthogonal direct sum of subspaces $\mathcal{H}_{k}$, $k \in \mathbb{Z}$, of form (9) is called quasi-orthogonal or $U$-orthogonal. If the operator $U$ is represented as $U=I+W$, where $W \in \mathfrak{S}_{2}(\mathcal{H})$, then the quasi-orthogonal expansion of the space $\mathcal{H}$ is called Riesz expansion.

We observe that $U$-orthogonal expansion (11) of the space $\mathcal{H}$ is called orthogonal if in $\mathcal{H}$ we introduce an equivalent scalar product of form

$$
(x, y)_{*}=(U x, U y), \quad x, y \in \mathcal{H} .
$$

Definition 10. We shall say that a linear closed operator $A: D(A) \subset \mathcal{H} \rightarrow \mathcal{H}$ is a quasiorthogonal (U-orthogonal) direct sum of bounded operators $\widetilde{A}_{k}, k \in \mathbb{Z}$, with respect to the quasi-orthogonal expansion of space $\mathcal{H}$ of form (11) if $\widetilde{A}_{k}=U A_{k} U^{-1}, k \in \mathbb{Z}$, for some invertible operator $U \in$ End $\mathcal{H}$. This is written as

$$
A=\bigoplus_{k \in \mathbb{Z}} \widetilde{A}_{k}
$$

We return back to the operator $L$ defined by formulae (5), (6). We represent it as $L=L_{0}-V$, where $L_{0}: D\left(L_{0}\right)=D(L) \subset L_{2} \rightarrow L_{2}$ is the operator of differentiating $L_{0}=\frac{d}{d s}$ and

$$
(V y)(s)=v(s) y(\omega-s) \text {. }
$$

The operator $V$ is well-defined thanks to the inclusion $D\left(L_{0}\right) \subset D(V)$. In what follows, the operator $L_{0}$ serves as an unperturbed operator, while the operator $V$ is regarded as a perturbation.

Let us describe the spectral property of the unperturbed operator $L_{0}$. The spectrum $\sigma\left(L_{0}\right)$ of the operator $L_{0}$ is represented as

$$
\sigma\left(L_{0}\right)=\cup_{k \in \mathbb{Z}} \sigma_{k},
$$

where $\sigma_{k}=\left\{\lambda_{k}\right\}, \lambda_{k}=\frac{i 2 \pi k}{\omega}, k \in \mathbb{Z}$, are the isolated eigenvalues. The corresponding eigenvectors are the functions $e_{k}(s)=e^{i \frac{2 \pi k}{\omega} s}, s \in[0, \omega], k \in \mathbb{Z}$, forming an orthonormalized basis in the spaces $L_{2}$ with respect to the introduced scalar product. The corresponding spectral projector $\mathcal{P}_{n}=P\left(\sigma_{n}, L_{0}\right), n \in \mathbb{Z}$, (Riesz projector) is defined by the formula

$$
\left(\mathcal{P}_{n} x\right)(s)=\frac{1}{\omega}\left(\int_{0}^{\omega} x(\tau) e^{-i \frac{2 \pi k}{\omega} \tau} d \tau\right) e^{i \frac{2 \pi k}{\omega} s}=\widehat{x}(n) e^{i \frac{2 \pi n}{\omega} s}, x \in L_{2}, s \in[0, \omega] .
$$

Here $\widehat{x}(n), n \in \mathbb{Z}$, is the Fourier coefficient of a periodic function $x \in L_{2}$ defined by the formula

$$
\widehat{x}(n)=\frac{1}{\omega} \int_{0}^{\omega} x(\tau) e^{-i \frac{2 \pi n}{\omega} \tau} d \tau .
$$

We observe that an unperturbed operator $L_{0}=\frac{d}{d s}$ is an orthogonal sum of the operators $\left(L_{0}\right)_{k}=L_{0} \mid \mathcal{H}_{k}=\frac{i 2 \pi k}{\omega} I_{k}$, where $I_{k}$ stands for the identity operator in the one-dimensional space $\mathcal{H}_{k}=\operatorname{Im} \mathcal{P}_{k}$, that is, $L_{0}=\oplus_{k \in \mathbb{Z}}\left(L_{0}\right)_{k}$. At that, all operators $\left(L_{0}\right)_{k}, k \in \mathbb{Z}$, have rank one 1 and are represented as $\left(L_{0}\right)_{k}=\lambda_{k} I_{k}, k \in \mathbb{Z}$. 
Let $\mathcal{P}_{(k)}=\sum_{|i| \leqslant k} \mathcal{P}_{i}, k \geqslant 0$. Then the operator $L_{0}$ is also an orthogonal direct sum of the operators

$$
L_{0}=\left(L_{0}\right)_{(k)} \oplus\left(\bigoplus_{|j|>k}\left(L_{0}\right)_{j}\right)=\left(L_{0}\right)_{(k)} \oplus\left(\bigoplus_{|j|>k} i \frac{2 \pi j}{\omega} I_{j}\right), \quad k \in \mathbb{Z}_{+}, \quad j \in \mathbb{Z},
$$

where $\left(L_{0}\right)_{(k)}$ is the restriction of the operator $L_{0}=\frac{d}{d s}$ on the subspace $\mathcal{H}_{(k)}=\operatorname{Im} \mathcal{P}_{(k)}$ with respect of the representation of the space $L_{2}$ as

$$
L_{2}=\mathcal{H}_{(k)} \oplus\left(\bigoplus_{|j|>k} \mathcal{H}_{j}\right)
$$

We note that $\left(L_{0}\right)_{(k)}=\bigoplus_{|j| \leqslant k} \frac{i 2 \pi j}{\omega} I_{j}$ with respect to the orthogonal expansion $\mathcal{H}_{(k)}=\bigoplus_{|j| \leqslant k} \mathcal{H}_{j}$.

The next theorem is a base of all results and estimates in the paper.

Theorem 3. There exists a number $k \in \mathbb{Z}_{+}=\mathbb{N} \cup\{0\}$ such that the operator $L=L_{0}-V$ is similar to the operator $L_{0}-V_{0}$, where the operator $V_{0}$ belongs to the space $\mathfrak{S}_{2}(\mathcal{H})$, the identity

$$
L U=U\left(L_{0}-V_{0}\right)
$$

holds true and the subspaces $\mathcal{H}_{(k)}=\operatorname{Im} \mathcal{P}_{(k)}$ and $\mathcal{H}_{j}=\operatorname{Im} \mathcal{P}_{j},|j|>k$, are invariant with respect to the operators $V_{0}, L_{0}$. Moreover, the operator $L$ is an $U$-orthogonal direct sum of form

$$
L=U\left(L_{0}-\left(V_{0(k)} \oplus \bigoplus_{|j|>k} V_{0 j}\right)\right) U^{-1}
$$

with respect to the Riesz expansion (U-orthogonal expansion) of the space $\mathcal{H}=U \mathcal{H}_{(k)} \oplus \bigoplus_{|j|>k} U \mathcal{H}_{j}$. The invertible operator of transformation $U$ in End $L_{2}$ is represented as $U=I+W$, where $W \in \mathfrak{S}_{2}(\mathcal{H})$.

A specific form of the operators $U$ and $V_{0}$ will be written out in Sections 3, 4, 5 .

Theorem 4. The spectrum of the operator $L$ can be represented as

$$
\sigma(L)=\widetilde{\sigma}_{(k)} \cup\left(\bigcup_{|j|>k} \widetilde{\sigma}_{j}\right),
$$

where the set $\widetilde{\sigma}_{(k)}$ contains at most $2 k+1$ eigenvalues, the sets $\widetilde{\sigma}_{j},|j|>k$, consist of a single point and $\widetilde{\sigma}_{j}=\left\{\widetilde{\lambda}_{j}\right\}, \widetilde{\lambda}_{j}=i \frac{2 \pi j}{\omega}-b_{j}$, and $\sum_{|j|>k}\left|b_{j}\right|^{2}<\infty$.

The associated eigenvectors $\widetilde{e}_{n}$ form a Bari basis in the space $L_{2}$ (in particular, Riesz basis) and the estimate holds:

where $\sum_{|n|>m} b_{1 n}^{2}<\infty$.

$$
\left\|\widetilde{e}_{n}-e_{n}\right\|=b_{1 n}, \quad|n|>m
$$

In work [5], there were given more specified formulae for the eigenvalues and eigenvectors of the operator $L$ in the case of a smooth on the segment $[0,1]$ potential $v$ such that $v(0)=v(1)$.

In paper [17] there were obtained the estimates for the weighted means of the eigenvalues in the sets $\sigma_{n},|n|>m$, and the estimates for the deviations $\operatorname{dist}\left(i \frac{2 \pi j}{\omega}, \widetilde{\sigma}_{j}\right)$.

We note that the properties of differential operators with involution discussed in the present work differs significantly from the spectral properties of differential operators [31, [32].

The next theorem is formulated under the assumptions of Theorems 3 and 4 in terms of notations introduced in Theorem 4 . 
Let $\widetilde{\mathcal{P}}_{(k)}=P\left(\widetilde{\sigma}_{(k)}, L\right), k \geqslant 0, \widetilde{\mathcal{P}}_{n}=P\left(\widetilde{\lambda}_{n}, L\right),|n|>m$, be the spectral projectors constructed by the spectral sets $\widetilde{\sigma}_{(k)}$ and $\widetilde{\sigma}_{j},|j|>k$, respectively.

Theorem 5. The limiting relation

$$
\lim _{n \rightarrow \infty}\left\|\widetilde{\mathcal{P}}_{(k)}+\sum_{|j|=k+1}^{n} \widetilde{\mathcal{P}}_{j}-\mathcal{P}_{(k)}-\sum_{|j|=k+1}^{n} \mathcal{P}_{j}\right\|_{2}=0
$$

holds true.

The next theorem strengthens essentially the result of Theorem 1 .

Theorem 6. The differential operator $L$ is a generator of a strongly continuous group of operators

$$
T: \mathbb{R} \rightarrow \text { End } L_{2} .
$$

The group $T: \mathbb{R} \rightarrow$ End $L_{2}$ is similar to the group $\widetilde{T}: \mathbb{R} \rightarrow$ End $L_{2}$, which admits an orthogonal expansion of form

$$
\widetilde{T}(t)=\bigoplus_{j=-\infty}^{-k-1} e^{\left(\frac{i 2 \pi j}{\omega}-b_{j}\right) I_{j} t} \oplus e^{B_{(k)} t} \bigoplus_{j=k+1}^{\infty} e^{\left(\frac{i 2 \pi j}{\omega}-b_{j}\right) I_{j} t}, \quad t \in \mathbb{R}
$$

with respect to the expansion of the space $L_{2}$ of form (14) for some integer $k \geqslant 0$, and $T(t)=$ $U \widetilde{T}(t) U^{-1}, t \in \mathbb{R}$. Moreover, the operator of transformation $U \in$ End $L_{2}$ of the group $\widetilde{T}$ into the group $T$ can be represented as $U=I+W$, where $W \in \mathfrak{S}_{2}(\mathcal{H})$. The operator $B_{(k)}$ belongs to End $\mathcal{H}_{(k)}$ and $\sum_{|j|>k}\left|b_{j}\right|^{2}<\infty$.

Thus, the group of operators $T(t), t \in \mathbb{R}$, admits an $U$-orthogonal expansion with respect to an $U$-orthogonal expansion of the space $L_{2}$ of form (11). The number $k$ in Theorem 6 will be found later in Section 4, see Theorem 16 .

Let $\psi=U^{-1} \varphi$ and the function $\psi$ is expanded into the Fourier series

$$
\psi(s)=\sum_{n \in \mathbb{Z}} \widehat{\psi}(n) e^{i \frac{2 \pi n}{\omega} s}, \quad s \in[0, \omega]
$$

It is obvious that $\mathcal{P}_{j} \psi=\widehat{\psi}(j) e_{j}, j \in \mathbb{Z}$.

Definition 11. The representation of the group of operators (mild solutions) of form

$$
T(t) \varphi=U \widetilde{T}(t) U^{-1} \varphi=\sum_{j=-\infty}^{-k-1} e^{\left(\frac{i 2 \pi j}{\omega}-b_{j}\right) t} U \mathcal{P}_{j} \psi+U e^{B_{(k)} t} \mathcal{P}_{(k)} \psi+\sum_{j=k+1}^{\infty} e^{\left(\frac{i 2 \pi j}{\omega}-b_{j}\right) t} U \mathcal{P}_{j} \psi
$$

is called the Fourier series of the mild solution $u(t, s)=(T(t) \varphi)(s), t \in \mathbb{R}, s \in[0, \omega], \varphi \in L_{2}$, to problem (1).

Representation (15) of Theorem 6 implies immediately the next theorem.

Theorem 7. The group $\widetilde{T}$ can be represented as

$$
\widetilde{T}(t)=e^{B_{(k)} t} \mathcal{P}_{(k)}+\sum_{|j| \geqslant k+1} e^{\left(i \frac{2 \pi j}{\omega}-b_{j}\right) t} \mathcal{P}_{j}
$$

We consider sequences of the numbers $\beta_{l}=\left\|\mathcal{P}_{l} W\right\|_{2}, \alpha_{l}=\sup _{|j| \geqslant l}\left|b_{j}\right|, l \in \mathbb{Z}$. They possess the properties $\lim _{l \rightarrow \infty} a_{l}=0$ and $\left(\beta_{l}\right)$ is a square integrable sequence.

The representation of solution as (16) implies the following theorem. 
Theorem 8. For each function $\varphi \in L_{2}$ the estimates hold:

$$
\begin{aligned}
& \left\|T(t) \varphi-U e^{B_{(k)} t} \mathcal{P}_{(k)} \psi-\sum_{k \leqslant|j| \leqslant N} e^{\left(\frac{i 2 \pi j}{\omega}-b_{j}\right) t} U \mathcal{P}_{j} \psi(j)\right\|_{2} \\
& \leqslant C\left(\sum_{|j| \geqslant N+1} e^{2 b_{j}|t|}\left(|\widehat{\psi}(j)|^{2}+\beta_{j}^{2}\|\psi\|^{2}\right)\right)^{\frac{1}{2}} \leqslant C e^{\alpha_{N+1}|t|}\left(\sum_{|j| \geqslant N+1}\left(|\widehat{\psi}(j)|^{2}+\beta_{j}^{2}\|\psi\|^{2}\right)\right)^{\frac{1}{2}}, \quad t \in \mathbb{R},
\end{aligned}
$$

for $N>k$ and some constant $C>0$.

The presence of the group of operators $T$ allows us to well define the operator $\frac{d}{d t}-L$ in a series of functional spaces. B the symbol $C_{b}=C_{b}\left(\mathcal{J}, L_{2}\right)$ we denote a Banach space of continuous bounded on $\mathcal{J}$ functions with values in $L_{2}$ and the norm $\|x\|_{\infty}=\sup _{t \in \mathcal{J}}\|x(t)\|_{2}, x \in C_{b}$. It follows from Theorem 4 that $\sigma(L) \cap i \mathbb{R}$ is at most countable set having no finite accumulation points. This is [33] implies immediately the next theorem.

Theorem 9. Each mild bounded solution $\widetilde{u} \in C_{b}$ of problem (1) is a Bohr almost periodic function (see [34]).

Hereinafter till the end of the section we assume that $\mathcal{J}$ coincides either with $\mathbb{R}_{+}$or with $\mathbb{R}$.

Definition 12 ([35], [36]). A function $x_{0} \in C_{b}$ is called slowly varying at infinity if $\lim _{|s| \rightarrow \infty}\left\|x_{0}(t+s)-x_{0}(s)\right\|_{2}=0$.

Definition 13 ([35], [36]). A uniformly continuous function $x \in C_{b}$ is called almost periodic at infinity if for each $\varepsilon>0$ there exists a generalized trigonometric polynomial of form $\varphi_{n}(t)=$ $\sum_{k=1}^{n} x_{k}(t) e^{i \lambda_{k} t}, t \in \mathcal{J}$, where $x_{k}, 1 \leqslant k \leqslant n$, are slowly varying at infinity functions and $\lambda_{k}$, $1 \leqslant k \leqslant n$, are real numbers such that

$$
\sup _{t \in \mathcal{J}}\left\|x(t)-\sum_{k=1}^{n} x_{k}(t) e^{i \lambda_{k} t}\right\|<\varepsilon .
$$

By $C_{0}=C_{0}\left(\mathcal{J}, L_{2}\right)$ we denote the space of functions vanishing at infinity, that is, $\lim _{|t| \rightarrow \infty}\|x(t)\|_{2}=0, x \in C_{0}$. Theorem 6.3 in 35] implies immediately the following theorem.

Theorem 10. Let $\widetilde{u} \in C_{0}$ be a mild solution to inhomogeneous problem (2) and $\tilde{f} \in C_{0}$. Then $\widetilde{u}: \mathcal{J} \rightarrow L_{2}$ is almost periodic at infinity.

\section{Method OF SIMILAR OPERATORS}

We observe that the method of similar operator is usually employed in the spectral analysis of various classes of differential (see 37] - 40]) and difference (see [41, [42]) operators. While presenting the method, we shall base on works [21], [22].

Let $A: D(A) \subset \mathcal{H} \rightarrow \mathcal{H}$ be a linear closed operator with a dense domain acting in a complex (abstract) Hilbert space $\mathcal{H}$. We introduce a linear space $\mathfrak{L}_{A}(\mathcal{H})$ of operators acting in $\mathcal{H}$ and relatively bounded with respect to the operator $A: D(A) \subset \mathcal{H} \rightarrow \mathcal{H}$. We shall say that an operator $B: D(B) \subset \mathcal{H} \rightarrow \mathcal{H}$ is relatively bounded with respect to the operator $A$ if $D(B) \supset D(A)$ and the quantity $\|B\|_{A}=\inf \{C>0:\|B x\| \leqslant C(\|x\|+\|A x\|), x \in D(A)\}$ is finite. This quantity is chosen to be the norm of the operator $B \in \mathfrak{L}_{A}(\mathcal{H})$ in $\mathfrak{L}_{A}(\mathcal{H})$. Thus, $\mathfrak{L}_{A}(\mathcal{H})$ is a Banach space.

We consider a perturbed abstract operator $A-B: D(A) \subset \mathcal{H} \rightarrow \mathcal{H}$, where $B \in \mathfrak{L}_{A}(\mathcal{H})$, and the spectral properties of a linear closed operator $A$ are known. 
We define a transformer (that is, a linear operator in spaces of operators; a terminology due to M.G. Krein) $\operatorname{ad}_{A}: D\left(\operatorname{ad}_{A}\right) \subset$ End $\mathcal{H} \rightarrow$ End $\mathcal{H}$ by the formula

$$
\operatorname{ad}_{A} X=A X-X A, \quad X \in D\left(\operatorname{ad}_{A}\right),
$$

with domain $D\left(\operatorname{ad}_{A}\right)$ formed by operators $X \in \operatorname{End} \mathcal{H}$ possessing the properties:

1) $X D(A) \subset D(A)$;

2) the operator $\operatorname{ad}_{A} X: D(A) \rightarrow \mathcal{H}$ admits a bounded extension $Y$ on $\mathcal{H}$ and we let $\operatorname{ad}_{A} X=Y$ (such extension is unique).

The most important notion of the method of similar operator is the notion of an admissible triple.

Definition $14([21],[22])$. Let $\mathcal{M}$ be a linear subspace in $\mathfrak{L}_{A}(\mathcal{H})$ and $J: \mathcal{M} \rightarrow \mathcal{M}, \Gamma$ : $\mathcal{M} \rightarrow$ End $\mathcal{H}$ be transformers. A triple $(\mathcal{M}, J, \Gamma)$ is called an admissible triple for the operator $A$, and $\mathcal{M}$ is called a space of admissible perturbations if

1) $\mathcal{M}$ is a Banach space with its norm $\|\cdot\|_{*}$ continuously embedded into $\mathfrak{L}_{A}(\mathcal{H})$, that is, there exists a constant $C>0$ such that $\|X\|_{A} \leqslant C\|X\|_{*}$ for each $X \in \mathcal{M}$;

2) $J$ and $\Gamma$ are bounded linear operator and $J$ is a projector;

3) $(\Gamma X) D(A) \subset D(A)$ and the identities

$$
\left(\operatorname{ad}_{A} \Gamma X\right) x=(X-J X) x, \quad x \in D(A), \quad X \in \mathcal{M},
$$

hold and $\Gamma X \in$ End $\mathcal{H}$ is the unique solution to the equation

$$
\operatorname{ad}_{A} Y=A Y-Y A=X-J X
$$

satisfying the condition $J Y=0$;

4) $X \Gamma Y,(\Gamma X) Y \in \mathcal{M}$ for all $X, Y \in \mathcal{M}$ and there exists a constant $\gamma>0$ such that

$$
\|\Gamma\| \leqslant \gamma, \quad \max \left\{\|X \Gamma Y\|_{*},\|(\Gamma X) Y\|_{*}\right\} \leqslant \gamma\|X\|_{*}\|Y\|_{*} ;
$$

5) $J((\Gamma X) J Y)=0$ for all $X, Y \in \mathcal{M}$;

6) for all $X \in \mathcal{M}$ and $\varepsilon>0$ there exists a number $\lambda_{\varepsilon} \in \rho(A)$ such that $\left\|X\left(A-\lambda_{\varepsilon} I\right)^{-1}\right\|_{\infty}<\varepsilon$.

Let is fox an admissible triple $(\mathcal{M}, J, \Gamma)$ for an unperturbed operator $A$.

Theorem 11 ([21], [22]). Let $(\mathcal{M}, J, \Gamma)$ be an admissible triple for the operator $A: D(A) \subset$ $\mathcal{H} \rightarrow \mathcal{H}$ and $B$ be some operator in $\mathcal{M}$. If

$$
4\|J\|\|B\|_{*} \gamma<1
$$

the operator $A-B$ is similar to the operator $A-J X_{*}$, where $X_{*} \in \mathcal{M}$ solves the nonlinear equation

$$
X=B \Gamma X-(\Gamma X)(J B)-(\Gamma X) J(B \Gamma X)+B=\Phi(X) .
$$

$A$ solution $X_{*}$ can be found by the method of simple iterations letting $X_{0}=0, X_{1}=B, \ldots$ The similarity transformation of the operator $A-B$ into the operator $A-J X_{*}$ is made by the invertible operator $I+\Gamma X_{*} \in$ End $\mathcal{H}$. The mapping $\Phi: \mathcal{M} \rightarrow \mathcal{M}$ is contracting in the ball $\left\{X \in \mathcal{M}:\|X-B\|_{*} \leqslant 3\|B\|_{*}\right\}$.

Lemma 1 and Theorem 11 imply the following theorem; in its formulation we employ the notations of Theorem 11.

Theorem $12([22])$. Let $(\mathcal{M}, J, \Gamma)$ be an admissible triple for the operator $A: D(A) \subset \mathcal{H} \rightarrow$ $\mathcal{H}$, the operator $B \in \mathcal{M}$ satisfies condition (17) and the operator $A-J X_{*}$ is a generator of a strongly continuous group of operators $\widetilde{T}: \mathbb{R} \rightarrow$ End $\mathcal{H}$. Then the operator $A-B$ is a generator of a group of operators $T: \mathbb{R} \rightarrow$ End $\mathcal{H}$, defined by identities:

$$
T(t)=\left(I+\Gamma X_{*}\right) \widetilde{T}(t)\left(I+\Gamma X_{*}\right)^{-1}, \quad t \geqslant 0,
$$

where $X_{*}$ is a solution to equation (18). 
Quite often it is difficult to construct the space of admissible perturbations containing a considered perturbation. In this case one can make a preliminary similarity transformation of the considered operator, whose perturbation belongs to the space of admissible perturbations $\mathcal{M}$ in some admissible triple $(\mathcal{M}, J, \Gamma)$. Such transformation is possible under the assumptions of the next proposition.

Assumption 1 ([22]). Given an operator $C \in \mathfrak{L}_{A}(\mathcal{H})$, there exist operators $\Gamma C, J C \in$ $\mathfrak{L}_{A}(\mathcal{H})$ satisfying the conditions:

1) $\Gamma C \in$ End $\mathcal{H}$ and $\|\Gamma C\|<1$;

2) $(\Gamma C) D(A) \subset D(A)$;

3) $C \Gamma C,(\Gamma C) J C \in \mathcal{M}$;

4) $A(\Gamma C) x-(\Gamma C) A x=C x-(J C) x, x \in D(A)$;

5) for each $\varepsilon>0$ there exists a number $\lambda_{\varepsilon} \in \rho(A)$ such that $\left\|C\left(A-\lambda_{\varepsilon} I\right)^{-1}\right\|<\varepsilon$.

Theorem 13 ([22]). Under the assumptons of Proposition 1, the operator $A-C$ is similar to the operator $A-J C-C_{0}$, where $C_{0}=(I+\Gamma C)^{-1}(C \Gamma C-(\Gamma C) J C)$, and the identity holds:

$$
(A-C)(I+\Gamma C)=(I+\Gamma C)\left(A-J C-C_{0}\right) .
$$

Let an unperturbed operator $A: D(A) \subset \mathcal{H} \rightarrow \mathcal{H}$ be a skew-adjoint operator and its spectrum $\sigma(A)$ admits the representation of form

$$
\sigma(A)=\bigcup_{k \in \mathbb{Z}} \Delta_{k}
$$

where $\Delta_{k}, k \in \mathbb{Z}$, are compact mutually disjoint sets. Let $P_{k}, k \in \mathbb{Z}$, be the Riesz projector constructed by the spectral set $\Delta_{k}, k \in \mathbb{Z}$. Then the system of projectors $\left\{P_{k}, k \in \mathbb{Z}\right\}$ and system of subspaces $\mathcal{H}_{n}=\operatorname{Im} P_{n}, n \in \mathbb{Z}$, satisfy the properties provided after Lemma 1 .

Let us fix an admissible triple $(\mathcal{M}, J, \Gamma)$ for the operator $A$, in which the transformer $J$ : $\mathcal{M} \rightarrow \mathcal{M}$ is well-defined by means of the formula

$$
J X=\sum_{k \in \mathbb{Z}} P_{k} X P_{k}, \quad X \in \mathcal{M}
$$

Thus, each of the operators $J X, X \in \mathcal{M}$, is an orthogonal direct sum of the operators $X_{k}=$ $P_{k} X \mid \mathcal{H}_{k}, X_{k} \in$ End $\mathcal{H}_{k}, k \in \mathbb{Z}$. Moreover, and this is very important, the operator $A-J X$ possesses the same property and

$$
A-J X=\bigoplus_{k \in \mathbb{Z}}\left(A_{k}-X_{k}\right)
$$

where $A_{k}=A \mid \mathcal{H}_{k}$ is the restriction of the operator $A$ on $\mathcal{H}_{k}$.

Theorem 14. Under the assumptions of Theorem 12, the operator $A-B$, where $B \in \mathcal{M}$, is similar to the operator $A-J X_{*}, X_{*} \in \mathcal{M}$, being an orthogonal direct sum of the operators (19), where $X=X_{*}$, with respect to the orthogonal expansion of the space $\mathcal{H}$ of form (9), where $\mathcal{H}_{i}=\operatorname{Im} P_{i}, i \in \mathbb{Z}$. The operator $A-B$ is an quasi-orthogonal direct sum of bounded operators with respect to the quasi-orthogonal expansion of the space $H$ of form (9), where $U$ is an operator of transformation of the operator $A-B$ into the operator $A-J X_{*}$.

We denote the operator $A-J X_{*}$ by $A_{0}$. For operators $A_{0}$ of form $(19)$ the following theorem holds.

Lemma 2. An operator $A_{0}$ of form (19) is a generator of some strongly continuous group $T_{0}: \mathbb{R} \rightarrow$ End $\mathcal{H}$, if and only if the condition

$$
\sup _{|t| \leqslant b u p}\left\|e^{A_{0, n} t}\right\|_{\text {End } \mathcal{H}_{n}}=C(b)<\infty,
$$


holds, where $b \geqslant 1$. If condition (20) holds, the operator $T_{0}(t), t \in \mathbb{R}$, are represented as an orthogonal direct sum

$$
T_{0}(t)=\bigoplus_{n \in \mathbb{Z}} e^{A_{0, n} t}, \quad t \in \mathbb{R}
$$

with respect to the expansion of the space $\mathcal{H}$ of form (9).

Proof. If condition (20) holds, the formula

$$
T_{0}(t) x=\sum_{n \in \mathbb{Z}} e^{A_{0, n} t} P_{n} x, \quad t \in \mathbb{R},
$$

defines a bounded operator that is due to the Parseval identity and the estimate

$$
\left\|T_{0}(t) x\right\|^{2}=\sum_{n \in \mathbb{Z}}\left\|e^{A_{0, n} t} P_{n} x\right\|^{2} \leqslant C^{2}(b) \sum_{n \in \mathbb{Z}}\left\|P_{n} x\right\|^{2}=C^{2}(b)\|x\|^{2}, \quad x \in \mathcal{H}, \quad|t| \leqslant b .
$$

It is straightforward to check that the operators $T_{0}(t) \in$ End $\mathcal{H}, t \in \mathbb{R}$, form a group of operators. Since this group is strongly continuous on a dense subset of vectors represented as $x=\sum_{|n| \leqslant m} P_{n} x, m \in \mathbb{Z}_{+}$, it is strongly continuous on the entire space $\mathcal{H}$.

The inverse statement is obvious. The proof is complete.

Thus, the construction of the group of operators for the initial operator is reduced to constructing a group of operators for the operator being an orthogonal direct sum of operators with respect to expansion (19) by using Lemma 2.

\section{FiRST TRANSFORMATION OF SIMILARITY}

In what follows as the space of admissible perturbations $\mathcal{M}$ in the method of similar operators, the space $\mathfrak{S}_{2}\left(L_{2}\right)$ will serve, while as a system of projectos $\mathcal{P}=\left(\mathcal{P}_{n}\right), n \in \mathbb{Z}$, we choose a system of spectral projectors of an unperturbed operator $L_{0}$ (see formula (13)).

Hereinafter the symbol $\mathcal{H}$ stands for a Hilbert space $L_{2}$.

In what follows we employ the matrices of the operators of two forms: operator and scalar ones. To each operator $X \in$ End $\mathcal{H}$ we associate an operator matrix $X \sim\left(X_{i j}\right)$, where $X_{i j}=$ $\mathcal{P}_{i} X \mathcal{P}_{j} \in$ End $\mathcal{H}, i, j \in \mathbb{Z}$, and the projectors $\mathcal{P}_{i}, i \in \mathbb{Z}$, are defined by formula (13).

The scalar matrix $X \sim\left(x_{i j}\right)$ consists of the entries $x_{i j}, i, j \in \mathbb{Z}$, defined by the formula $x_{i j}=\left(X e_{j}, e_{i}\right)$, and as a basis $e_{i}, i \in \mathbb{Z}$, we choose normalized eigenvectors of the unperturbed operator $L_{0}$.

In what follows we employ the following properties of the ideal $\mathfrak{S}_{2}(\mathcal{H})$ (see [1]):

1) The operator $X \in$ End $\mathcal{H}$ is a Hilbert-Schmidt operator if and only if its matrix $x_{k n}=$ $\left(X g_{n}, g_{k}\right), n, k \in \mathbb{Z}$, is a Hilbert-Schmidt matrix for some orthonormalized basis $\left\{g_{k}, k \in \mathbb{Z}\right\}$ and $\|X\|_{2}^{2}=\sum_{k, n \in \mathbb{Z}}\left|x_{k n}\right|^{2}$.

2) The product $X Y$ of the operators $X, Y \in \mathfrak{S}_{2}(\mathcal{H})$ is a nuclear operator and $\|X Y\|_{1} \leqslant$ $\|X\|_{2}\|Y\|_{2}$.

3) Let $\left\{Q_{n}, n \geqslant 0\right\}$ be the system of orthoprojectors in End $\mathcal{H}$ forming the partition of the unity. Then $\|X\|_{2}^{2}=\sum_{n, m=0}^{\infty}\left\|Q_{n} X Q_{m}\right\|_{2}^{2}$.

4) Let the operator $X: D(X) \subset \mathcal{H} \rightarrow \mathcal{H}$ belongs the space $\mathfrak{L}_{A}(\mathcal{H})$ and hence, it has a domain $D(X)$ dense in $\mathcal{H}$. If the quantity $\sum_{n, k \in \mathbb{Z}}\left|\left(X e_{n}, e_{k}\right)\right|^{2}$ is finite for some orthonormalized basis $\left\{e_{k}, k \in \mathbb{Z}\right\}$ with the property $e_{k} \in D(X), k \in \mathbb{Z}$, then the operator $X$ has the unique extension on $\mathcal{H}$. This extension is a Hilbert-Schmidt operator and it is denoted by the same symbol $X$. 
The notion of an operator matrix is naturally extended to the operators relatively bounded with respect to the operator $L_{0}$, not necessarily bounded.

To each operator $X: D(A) \subset L_{2} \rightarrow L_{2}, X \in \mathfrak{L}_{L_{0}}(\mathcal{H})$, we associate an operator matrix $\left(X_{i j}\right)$, $i, j \in \mathbb{Z}$, formed by bounded operator $X_{i j}=\mathcal{P}_{i} X \mathcal{P}_{j} \in$ End $\mathcal{H}, i, j \in \mathbb{Z}$.

We also note that in the considered case $\left(\operatorname{dim} \operatorname{Im} P_{j}=1\right.$ for all $\left.j \in \mathbb{Z}\right)$ :

$$
X_{i j} x=\left(P_{i} X P_{j}\right) x=\left(X P_{j} x, e_{i}\right) e_{i}=\left(X e_{i}, e_{j}\right)\left(x, e_{j}\right) e_{i}=\left(x_{i j}\right) \widehat{x}(j) e_{i}, \quad i, j \in \mathbb{Z} .
$$

The operator $V$ defined by formula $(12)$ is relatively bounded with respect the operator $L_{0}$ and it has the matrix $\left(V_{k n}\right)$ with respect the partition of the unity $\left(\mathcal{P}_{k}, k \in \mathbb{Z}\right)$, whose elements are defined by the formulae

$$
\left(V_{l n} x\right)(s)=\left(V e_{l}, e_{n}\right) \widehat{x}(n) e^{i \frac{2 \pi l}{\omega} s}=\frac{1}{\omega} \int_{0}^{\omega} v(\tau) e^{-i \frac{2 \pi l}{\omega}(\omega-\tau)} d \tau \cdot \widehat{x}(n) e^{i \frac{2 \pi l}{\omega} s}=\widehat{v}(l+n) \widehat{x}(n) e^{i \frac{2 \pi l}{\omega} s} .
$$

Thus, the operator matrix of the operator $V$ is of the form

$$
V \sim\left(\begin{array}{cccccc}
\ldots & \ldots & \ldots & \ldots & \ldots & \ldots \\
\ldots & \widehat{v}(-2) & \widehat{v}(-1) & \widehat{v}(0) & \widehat{v}(1) & \ldots \\
\ldots & \widehat{v}(-1) & \widehat{v}(0) & \widehat{v}(1) & \widehat{v}(2) & \ldots \\
\ldots & \widehat{v}(0) & \widehat{v}(1) & \widehat{v}(2) & \widehat{v}(3) & \ldots \\
\ldots & \widehat{v}(1) & \widehat{v}(2) & \widehat{v}(3) & \widehat{v}(4) & \ldots \\
\ldots & \ldots & \ldots & \ldots & \ldots & \ldots
\end{array}\right)
$$

In what follows by $\mathcal{P}_{(m)}$ we denote the projector $\mathcal{P}_{(m)}=\sum_{|i| \leqslant m} \mathcal{P}_{i}$. We introduce operators $J_{m} V, \Gamma_{m} V, m \geqslant 0$, by the formulae

$$
\begin{aligned}
& J V=J_{0} V=\sum_{i \in \mathbb{Z}} \mathcal{P}_{i} V \mathcal{P}_{i}, \\
& J_{m} V=\mathcal{P}_{(m)} V \mathcal{P}_{(m)}+\sum_{|i|>m} \mathcal{P}_{i} V \mathcal{P}_{i}, \quad m \geqslant 0, \\
& \Gamma V=\Gamma_{0} V=\frac{\omega}{2 \pi i} \sum_{n \in \mathbb{Z} \backslash\{0\}} \widetilde{V}_{n}, \quad \widetilde{V}_{n}=\sum_{k-j=n} \frac{\mathcal{P}_{k} V \mathcal{P}_{j}}{k-j}, \\
& \Gamma_{m} V=\Gamma V-\mathcal{P}_{(m)}(\Gamma V) \mathcal{P}_{(m)} .
\end{aligned}
$$

The matrices of the operators $J V$ and $\Gamma V$ with respect the partition of the unity $\left(\mathcal{P}_{k}, k \in \mathbb{Z}\right)$ are of the form

$$
\begin{gathered}
J V \sim\left(\begin{array}{ccccc}
\cdots & \ldots & \ldots & \ldots & \ldots \\
\ldots & \widehat{v}(-2) & 0 & 0 & \ldots \\
\ldots & 0 & \widehat{v}(0) & 0 & \ldots \\
\ldots & 0 & 0 & \widehat{v}(2) & \ldots \\
\ldots & \ldots & \ldots & \ldots & \ldots
\end{array}\right), \\
\Gamma V \sim \frac{\omega}{2 \pi i}\left(\begin{array}{cccccc}
\ldots & \ldots & \ldots & \ldots & & \\
\ldots & 0 & -\widehat{v}(-1) & -\frac{1}{2} \widehat{v}(0) & -\frac{1}{3} \widehat{v}(1) & \ldots \\
\ldots & \widehat{v}(-1) & 0 & -\widehat{v}(1) & -\frac{1}{2} \widehat{v}(2) & \ldots \\
\ldots & \frac{1}{2} \widehat{v}(0) & \widehat{v}(1) & 0 & -\widehat{v}(3) & \ldots \\
\ldots & \frac{1}{3} \widehat{v}(1) & \frac{1}{2} \widehat{v}(2) & \widehat{v}(3) & 0 & \ldots \\
\ldots & \ldots & \ldots & \ldots & \ldots & \ldots
\end{array}\right) .
\end{gathered}
$$

The definition of the operators $J_{m} V$ and $\Gamma_{m} V, m \geqslant 0$, imply immediately that they belong to $\mathfrak{S}_{2}(\mathcal{H})$. They are well-defined, that is, the series defining these operators converge in $\mathfrak{S}_{2}(\mathcal{H})$. 
For the preliminary similarity transformation we shall also the operators $Z_{m}=V \Gamma_{m} V$, $m \geqslant 0$. The entries of the operator matrix of the operator $Z=V \Gamma V$ are defined by the formula

$$
Z_{i j}=\frac{\omega}{2 \pi i} \sum_{k \neq j} \frac{\widehat{v}(i+k) \widehat{v}(k+j)}{j-k}, \quad i, j \in \mathbb{Z} .
$$

Lemma 3. The operators $J_{m} V, \Gamma_{m} V$ and $V \Gamma_{m} V, m \geqslant 0$, are the operators in $\mathfrak{S}_{2}(\mathcal{H})$.

Proof. It remains to show that $Z \in \mathfrak{S}_{2}(\mathcal{H})$. This is implied by an integral representation of the operator $Z=V \Gamma V$ of form

$$
(Z y)(s)=\frac{1}{2 \omega} \int_{0}^{2 \omega} f\left(\frac{s-\tau}{2}\right) \widehat{v}\left(\frac{2 \omega-s-\tau}{2}\right) v(s) y(\tau) d \tau
$$

given in [17], [21]. Here the function $f$ is defined as $f(s)=i\left(s-\frac{\omega}{2}\right)$.

Let $L_{\infty}([0, \omega], \mathbb{C})$ be a Banach space of (classes of) functions with values in $\mathbb{C}$ and essentially bounded on $[0, \omega]$; the norm is $\|x\|_{\infty}=$ vrai $\sup _{s \in[0, \omega]}\|x(s)\|$.

Lemma 4. The operators $\Gamma_{m} V, m \in \mathbb{Z}_{+}$, possess the properties:

1) $\left(\Gamma_{m} V\right) D\left(L_{0}\right) \subset D\left(L_{0}\right)$;

2) $L_{0}\left(\Gamma_{m} V\right) x-\left(\Gamma_{m} V\right) L_{0} x=\left(V-J_{m} V\right) x, x \in D\left(L_{0}\right)$;

3) For each $\varepsilon>0$ there exists a number $\lambda_{\varepsilon} \in \rho\left(L_{0}\right)$ such that $\left\|V\left(L_{0}-\lambda_{\varepsilon} I\right)^{-1}\right\|_{2}<\varepsilon$.

Proof. Let $\lambda_{\varepsilon} \in \rho\left(L_{0}\right)$. We consider a sequence o projectors $Q_{(n)}=\sum_{|j| \leqslant n} P_{j}, n \geqslant 0$. For each vector $y \in \mathcal{H}$ the identities hold:

$$
\begin{aligned}
Q_{(n)} L_{0}\left(\Gamma_{m} V\right)\left(L_{0}-\lambda_{\varepsilon} I\right)^{-1} y & =Q_{(n)}\left(\Gamma_{m} V\right) L_{0}\left(L_{0}-\lambda_{\varepsilon} I\right)^{-1} y+ \\
& +Q_{(n)}\left(V-J_{m} V\right)\left(L_{0}-\lambda_{\varepsilon} I\right)^{-1} y=Q_{(n)} \mathcal{L} y
\end{aligned}
$$

where the operator $\mathcal{L}$ can be represented as

$$
\mathcal{L} y=\left(\Gamma_{m} V\right) L_{0}\left(L_{0}-\lambda_{\varepsilon} I\right)^{-1} y+\left(V-J_{m} V\right)\left(L_{0}-\lambda_{\varepsilon} I\right)^{-1} y
$$

The mentioned identities can be checked on the basis vectors. Since the operator $\mathcal{L}$ is an operator in End $\mathcal{H}$, the sequence of the operators in the right hand side converges to the operator $\mathcal{L}$ in the norm of the space End $\mathcal{H}$. The closedness of the operator $L_{0}$ implies that $\Gamma_{m} V x \in D\left(L_{0}\right)$ as $x \in D\left(L_{0}\right)$ and the identity

$$
\left(\Gamma_{m} V\right) L_{0}\left(L_{0}-\lambda_{\varepsilon} I\right)^{-1}=L_{0}\left(\Gamma_{m} V\right)\left(L_{0}-\lambda_{\varepsilon} I\right)^{-1}+\left(V-J_{m} V\right)\left(L_{0}-\lambda_{\varepsilon} I\right)^{-1}
$$

holds. Thus, properties 1) and 2) hold true.

It remains to check property 3). Let $Y=V\left(L_{0}-\lambda_{\varepsilon} I\right)^{-1}, \lambda_{\varepsilon} \in \rho(A)$. In this representation of the operator $Y$ we consider it as a produce of two operators $\left(L_{0}-\lambda_{\varepsilon} I\right)^{-1} \in$ End $\mathcal{H}, V_{\infty}$ : $L_{\infty}([0, \omega], \mathbb{C}) \rightarrow L_{2},\left(V_{\infty} x\right)(s)=(V x)(s), s \in[0, \omega], x \in L_{\infty}([0, \omega], \mathbb{C})$. It is clear that $\|V\|_{\infty}=$ $\|v\|_{L_{2}}$. It remains to prove that $\left\|\left(L_{0}-\lambda_{\varepsilon} I\right)^{-1}\right\|<\varepsilon /\|v\|_{L_{2}}$ for a number $\lambda_{\varepsilon}$ of form $\lambda_{\varepsilon}=k$ for sufficiently large $k \in \mathbb{N}$ and a given $\varepsilon>0$. For each function $x \in \mathcal{H}$ the representation

$$
\left(L_{0}-k I\right)^{-1} x=\sum_{l \in \mathbb{Z}} \frac{\widehat{x}(l) e^{i \frac{2 \pi l}{\omega} s}}{\frac{i 2 \pi l}{\omega}-k}
$$

holds. This is why

$$
\left\|\left(L_{0}-k I\right)^{-1} x\right\|_{L_{2}} \leqslant\left(\sum_{l \in \mathbb{Z}} \frac{1}{k^{2}+\left(\frac{2 \pi l}{\omega}\right)^{2}}\right)^{\frac{1}{2}}\|x\|_{2} \leqslant \frac{\varepsilon\|x\|_{2}}{\|V\|_{L_{2}}}
$$


for sufficiently large $k$.

Therefore, we have proved the property $\left\|\left(L_{0}-k I\right)^{-1} x\right\|_{2} \leqslant \varepsilon /\|v\|_{L_{2}}$. Thus,

$$
\left\|V\left(L_{0}-k I\right)^{-1}\right\| \leqslant\|v\|_{L_{2}}\left\|\left(L_{0}-k I\right)^{-1}\right\|<\varepsilon .
$$

The proof is complete.

In the proof the next theorem, being a main result of this section, we shall make use of the following lemma.

Lemma 5. The identity holds: $\lim _{m \rightarrow \infty}\left\|\Gamma_{m} V\right\|_{2}=\lim _{m \rightarrow \infty}\left\|\Gamma V-P_{(m)}(\Gamma V) P_{(m)}\right\|=0$.

Proof. Since the operator $\Gamma V$ is the Hilbert-Schmidt operator, the statement of the lemma follows immediately the definition of the orthoprojectors $P_{(m)}, m \geqslant 0$.

The obtained statements of the sequence of the operators $J_{m} V, \Gamma_{m} V, m \geqslant 0$, and Theorem 13 implies the following theorem.

Theorem 15. There exists a number $m \in \mathbb{Z}_{+}$such that $\left\|\Gamma_{m} V\right\|_{2}<1$, that is, the operator $I+\Gamma_{m} V$ is invertible and the operator $L_{0}-V$ is similar to the operator $L_{0}-\widetilde{V}$, where

$$
\widetilde{V}=J_{m} V+\left(I+\Gamma_{m} V\right)^{-1}\left(V \Gamma_{m} V-\left(\Gamma_{m} V\right) J_{m} V\right) \in \mathfrak{S}_{2}(\mathcal{H}, \mathcal{P}),
$$

and the identity holds:

$$
\left(L_{0}-V\right)\left(I+\Gamma_{m} V\right)=\left(I+\Gamma_{m} V\right)\left(L_{0}-\tilde{V}\right) .
$$

The operator $\widetilde{V}$ can be represented as

$$
\widetilde{V}=J_{m} V+V \Gamma_{m} V-\left(\Gamma_{m} V\right) J_{m} V+C=J V+V \Gamma V-(\Gamma V) J V+C_{1},
$$

where $C, C_{1} \in \mathfrak{S}_{1}(\mathcal{H})$.

Proof. It remains to prove formula (23). The representation

$$
\begin{aligned}
\left(I+\Gamma_{m} V\right)^{-1}\left(V \Gamma_{m} V-\left(\Gamma_{m} V\right) J_{m} V\right)= & \left(I+\Gamma_{m} V\right)^{-1}\left(\Gamma_{m} V\right)\left(V \Gamma_{m} V\right. \\
& \left.-\left(\Gamma_{m} V\right) J_{m} V\right)+V \Gamma_{m} V-\left(\Gamma_{m} V\right) J_{m} V
\end{aligned}
$$

and the fact that a product of two operators in $\mathfrak{S}_{2}(\mathcal{H})$ is an operator in $\mathfrak{S}_{1}(\mathcal{H})$ yield the first part of identity (23). Since the operators $V \Gamma_{m} V-V \Gamma V, J_{m} V-J V, \Gamma_{m} V-\Gamma V$ are of finite rank, they belong to $\mathfrak{S}_{1}(\mathcal{H})$ and the second part of formula 23 holds. The proof is complete.

\section{Construction of transformers $J_{k}$ And $\Gamma_{k}, k \geqslant 0, \operatorname{IN} \mathfrak{S}_{2}(\mathcal{H})$}

It follows from Theorem 15 that the studied differential operator with involution is similar to the operator $L_{0}-\widetilde{V}$, where the operator $\widetilde{V}$ defined by formula 22 belongs to $\mathfrak{S}_{2}(\mathcal{H})$. Below, see Section 5, we shall apply the method of similar operator to the operator $L_{0}-\widetilde{V}$, cf. Theorem 3. At that we shall employ essentially the sequences of transformers

$$
J_{k}, \Gamma_{k}: \mathfrak{S}_{2}(\mathcal{H}) \rightarrow \mathfrak{S}_{2}(\mathcal{H}), \quad k \geqslant 0
$$


For each operator $X \in \mathfrak{S}_{2}(\mathcal{H})$ we define these transformers by the following formulae:

$$
\begin{aligned}
& J X=J X_{0}=\sum_{n \in \mathbb{Z}} \mathcal{P}_{n} X \mathcal{P}_{n}, \\
& J_{k} X=P_{(k)} X P_{(k)}+\sum_{|j|>k} \mathcal{P}_{j} X \mathcal{P}_{j}, \quad k \geqslant 1, \\
& \Gamma X=\Gamma_{0} X=\frac{\omega}{2 \pi i} \sum_{\substack{n \in \mathbb{Z} \\
n \neq 0}} \widetilde{X}_{n}, \quad \text { where } \quad \widetilde{X}_{n}=\frac{1}{n} \sum_{p-j=n} X_{p j}, \\
& \Gamma_{k} X=\Gamma X-P_{(k)}(\Gamma X) P_{(k)}, \quad k \geqslant 1 .
\end{aligned}
$$

Here $X_{p j}=\mathcal{P}_{p} X \mathcal{P}_{j}, p, j \in \mathbb{Z}$, are matrix entries of the operator $X$.

The transformers $J_{k}, \Gamma_{k} \in \operatorname{End}\left(\mathfrak{S}_{2}(\mathcal{H})\right), k \geqslant 0$, are well-defined and all written series converge in $\mathfrak{S}_{2}(\mathcal{H})$.

Definition 15. The operator $X_{k}=\sum_{\substack{i, j \in \mathbb{Z} \\ i-j=k}} X_{i j}, k \in \mathbb{Z}$ is called a $k$ th diagonal of the operator matrix of the operator $X \in \mathfrak{S}_{2}(\mathcal{H})$.

Remark 2. An nth diagonal $X_{n}$ of an operator $X \in \mathfrak{S}_{2}(\mathcal{H})$ satisfy the formulae

$$
\left\|X_{n}\right\|_{2}^{2}=\sum_{\substack{i, j \in \mathbb{Z} \\ i-j=n}}\left\|X_{i j}\right\|^{2},
$$

at that,

$$
\|X\|_{2}^{2}=\sum_{n \in \mathbb{Z}}\left\|X_{n}\right\|_{2}^{2}
$$

In papers [17], [21] there was employed another approach used for determining the transformers $J$ and $\Gamma$ acting End $\mathcal{H}$; that approach was based on the representation theory. To describe this approach, we mention that the differentiation operator $L_{0}=\frac{d}{d s}$ is a generator of an $\omega$-periodic group $S: \mathbb{R} \rightarrow$ End $L_{2, \omega},(S(t) x)(s)=x(s+t), t, s \in \mathbb{R}, x \in L_{2, \omega}$. This group can be represented as

$$
S(t) x=\sum_{n \in \mathbb{Z}} e^{i \frac{2 \pi n}{\omega} t} P_{n} x .
$$

To each operator $X \in$ End $\mathcal{H}$ we associate an $\omega$-periodic strongly continuous operator-valued function

$$
t \mapsto S(t) X S(-t): \mathbb{R} \rightarrow \text { End } \mathcal{H} .
$$

At that, there arises a isometric representation of period $\omega$ :

$$
\widetilde{S}: \mathbb{R} \rightarrow \text { End }(\text { End } \mathcal{H}), \quad \widetilde{S}(t)=S(t) X S(-t), \quad t \in \mathbb{R} .
$$

Given a function $\widetilde{S}: \mathbb{R} \rightarrow$ End $($ End $\mathcal{H})$, we consider its Fourier series

$$
\widetilde{S}(t) x \sim \sum_{n \in \mathbb{Z}} X_{n} x e^{i \frac{2 \pi n}{\omega} t}, \quad x \in \mathcal{H}, \quad t \in \mathbb{R},
$$

where the operators $X_{n}$ read as

$$
X_{n} x=\frac{1}{\omega} \int_{0}^{\omega} S(t) X S(-t) e^{-i \frac{2 \pi n}{\omega} t} d t, \quad x \in \mathcal{H}, \quad n \in \mathbb{Z} .
$$

The series $\sum_{n \in \mathbb{Z}} X_{n}$ is called the Fourier series of the operator $X \in$ End $\mathcal{H}$ with respect to the group of the operators $\widetilde{S}$ and the operators $X_{n}, n \in \mathbb{Z}$ are called the Fourier coefficients of this 
operator (see [43], [44]). It is important to note that $X_{n}$ is the $n$th diagonal of the operator $X \in \mathfrak{S}_{2}(\mathcal{H})$ in the sense of definition 15 .

Remark 3. The group of isometries $\widetilde{S}$ can be considered with respect to the subspace $\mathfrak{S}_{2}(\mathcal{H})$ invariant for this group. In this case the Fourier coefficients $X_{n}, n \in \mathbb{Z}$, are also operators belonging to $\mathfrak{S}_{2}(\mathcal{H})$.

We note that according works [17] and [21], the transformers $J$ and $\Gamma$ can be defined by the formulae

$$
\begin{aligned}
& (J X) x=X_{0} x=\frac{1}{\omega} \int_{0}^{\omega} S(\tau) X S(-\tau) x d \tau, \quad x \in \mathcal{H}, \\
& (\Gamma X) x=\frac{1}{\omega} \int_{0}^{\omega} f(\tau) S(\tau) X S(-\tau) x d \tau, \quad x \in \mathcal{H},
\end{aligned}
$$

where $f: \mathbb{R} \rightarrow \mathbb{C}$ is an $\omega$-periodic function $f(s)=i\left(s-\frac{\omega}{2}\right), s \in[0, \omega]$, with the Fourier series of form $f(s) \sim \sum_{n \neq 0} \frac{\omega}{2 \pi n} e^{i \frac{2 \pi n}{\omega} s}$.

It is important to observe that the definition of the transformers $J$ and $\Gamma$ by integral representation coincide with the definition on matrix entries by (24) and (25).

The definition of the transformer $\Gamma: \mathcal{M} \rightarrow \mathcal{M}$ by $(26)$ implies the following lemma.

Lemma 6. The operator $\Gamma V$ belongs to $\mathfrak{S}_{2}(\mathcal{H})$ and is an integral operator of form

$$
(\Gamma V) x(s)=\frac{1}{2 \omega} \int_{0}^{2 \omega} f\left(\frac{\omega-s-\tau}{2}\right) v\left(\frac{\omega+s-\tau}{2}\right) x(\tau) d \tau, \quad x \in L_{2} .
$$

At that, $(\Gamma V) x \in L_{\infty}([0, \omega], H), x \in L_{2}$.

\section{SECOND SIMILARITY TRANSFORMATION}

In order to prove Theorem 3, we shall need another space of admissible perturbations $\mathcal{M} \subset$ $\mathfrak{S}_{2}(\mathcal{H})$. To describe it, for each non-zero operator $X \in \mathfrak{S}_{2}(\mathcal{H})$ we introduce a two-sided sequence of real numbers of form

$$
\alpha_{n}(X)=\|X\|_{2}^{-\frac{1}{2}} \max \left\{\left(\sum_{\substack{|k| \geqslant n \\ k \in \mathbb{Z}}}\left\|\mathcal{P}_{k} X\right\|_{2}^{2}\right)^{\frac{1}{4}},\left(\sum_{\substack{|k| \geqslant n \\ k \in \mathbb{Z}}}\left\|X \mathcal{P}_{k}\right\|_{2}^{2}\right)^{\frac{1}{4}}\right\}, n \in \mathbb{Z} .
$$

The sequence $\left(\alpha_{n}(X)\right), n \in \mathbb{Z}$, possesses the following properties:

1) $\alpha_{n}(X)=\alpha_{-n}(X), n \in \mathbb{Z}$;

2) $\lim _{|n| \rightarrow \infty} \alpha_{n}(X)=0, n \in \mathbb{Z}$

3) $\alpha_{n}(X) \leqslant 1$ for each $n \in \mathbb{Z}$;

4) $\alpha_{n}(X) \geqslant \alpha_{n+1}(X), n \geqslant 0$;

5) $\alpha_{n}(X) \neq 0$ for each $n \in \mathbb{Z}$ if $P_{(m)} X P_{(m)} \neq X$ for each $m \in \mathbb{Z}_{+}$;

6) the quantity

$$
\sum_{n \in \mathbb{Z}} \frac{\left\|X \mathcal{P}_{n}\right\|_{2}^{2}+\left\|\mathcal{P}_{n} X\right\|_{2}^{2}}{\left(\alpha_{n}(X)\right)^{2}}
$$

is finite.

As a perturbation of the operator $L_{0}$, the operator $\widetilde{V}$ in $\mathfrak{S}_{2}(\mathcal{H})$ defined by formula 22 in Theorem 15 will serve. Without loss of generality we can assume that $P_{(n)} \widetilde{V} P_{(n)} \neq 0$ for all $n \in \mathbb{Z}_{+}$. Otherwise the operator $L_{0}-\widetilde{V}$ is an orthogonal sum of operators of finite rank $\left.\left(L_{0}-\widetilde{V}\right)\right|_{\mathcal{H}_{(n)}}$ for some $n \geqslant 0$ and of the operator $\left.L_{0}\right|_{\mathcal{H}^{(n)}}$, where $\mathcal{H}^{(n)}=I-\mathcal{H}_{(n)}$. 
Thus, in what follows we consider the operator $L_{0}-\widetilde{V}$.

For each operator $X \in \mathfrak{S}_{2}(\mathcal{H})$ we consider a self-adjoint compact operator $F_{X}$ defined by the formula

$$
F_{X}=\sum_{n \in \mathbb{Z}} \alpha_{n}(X) \mathcal{P}_{n}
$$

We observe that $F_{X} \in$ End $\mathcal{H}$ is a function of a self-adjoint operator $L_{0}$ of form $F_{X}=f_{X}\left(L_{0}\right)$, where $f_{X}: \sigma\left(L_{0}\right) \rightarrow \mathbb{R}_{+}, f_{X}\left(\lambda_{n}\right)=\alpha_{n}(X), n \in \mathbb{Z}$, and $\left\|F_{X}\right\|_{\infty}=\max _{n \in \mathbb{Z}}\left|\alpha_{n}(X)\right|=1$.

To simplify the writing, we redenote the operator $F_{\widetilde{V}}$ by $F$.

We introduce the set of the operators $\mathcal{M}$ in $\mathfrak{S}_{2}(\mathcal{H})$ admitting the representation of form

$$
X=X_{l} F, \quad X=F X_{r},
$$

where $X_{l}, X_{r} \in \mathfrak{S}_{2}(\mathcal{H})$. We let $\|X\|_{\mathcal{M}}=\max \left\{\left\|X_{l}\right\|_{2},\left\|X_{r}\right\|_{2}\right\}$. It is obvious that $\|X\|_{2} \leqslant\|X\|_{\mathcal{M}}$, $X \in \mathcal{M}$.

The property Ker $F=0$ (cf. Property 5 of the sequence $\alpha_{n}(X)$ ) allows us to conclude that the introduced set of the operators $\mathcal{M}$ is a Banach space.

It is obvious that for each operator $X$ in $\mathfrak{S}_{2}(\mathcal{H})$ the representation holds:

$$
X=\left(\sum_{n \in \mathbb{Z}} \frac{1}{\alpha_{n}(X)} X \mathcal{P}_{n}\right) F_{X}=F_{X}\left(\sum_{n \in \mathbb{Z}} \frac{1}{\alpha_{n}(X)} \mathcal{P}_{n} X\right) \text {. }
$$

This is why the perturbation $\widetilde{V}$ is in the space $\mathfrak{S}_{2}(\mathcal{H})$.

Since $\mathcal{M} \subset \mathfrak{S}_{2}(\mathcal{H})$, the transformers $J_{k}$ and $\Gamma_{k}, k \geqslant 0$, are defined also for the operators in $\mathcal{M}$. Moreover, the subspace $\mathcal{M}$ is invariant with respect to $J_{k}$ and $\Gamma_{k}, k \geqslant 0$, and

$$
\begin{array}{ll}
J_{k}\left(X_{l} F\right)=\left(J_{k} X_{l}\right) F, & J_{k}\left(F X_{r}\right)=F\left(J_{k} X_{r}\right), \\
\Gamma_{k}\left(X_{l} F\right)=\left(\Gamma_{k} X_{l}\right) F, & \Gamma_{k}\left(F X_{r}\right)=F\left(\Gamma_{k} X_{r}\right),
\end{array}
$$

where $X_{r}, X_{l} \in \mathfrak{S}_{2}(\mathcal{H})$.

To estimate the norms $\left\|\Gamma_{k}(X F)\right\|_{2}$ and $\left\|\Gamma_{k}(F X)\right\|_{2}, X \in \mathfrak{S}_{2}(\mathcal{H})$, we consider two sequences $\left(\alpha_{n}^{\prime}\right), n \in \mathbb{N}$, and $\left(\widetilde{\alpha}_{n}\right), n \in \mathbb{N}$, defined by the formulae

$$
\alpha_{n}^{\prime}=\max _{\substack{|i| \geqslant n \\|j|<n}} \frac{\left|\alpha_{i}-\alpha_{j}\right|}{|i-j|}, \quad \widetilde{\alpha}_{n}=\frac{\omega}{2 \pi}\left(2 \alpha_{n}+\alpha_{n}^{\prime}\right), \quad n \in \mathbb{N} .
$$

The sequences $\left(\alpha_{n}^{\prime}\right)$ and $\left(\widetilde{\alpha}_{n}\right)$ belong to the space $c_{0}(N)$ of converging to zero sequences.

The next lemma is an analogue of Lemma 3 in [21].

Lemma 7. For all $k \in \mathbb{Z}_{+}$and each operator $X \in \mathfrak{S}_{2}(\mathcal{H})$ the estimates hold:

$$
\max \left\{\left\|\Gamma_{k}(X F)\right\|_{2},\left\|\Gamma_{k}(F X)\right\|_{2}\right\} \leqslant \widetilde{\alpha}_{k+1}\|X\|_{2} .
$$

Proof. Let $\mathcal{P}^{(k)}=I-\mathcal{P}_{(k)}, k \in \mathbb{Z}_{+}$, be the additional to $\mathcal{P}_{(k)}$ projector. Then

$$
\left\|F \mathcal{P}^{(k)}\right\|_{\infty}=\left\|\sum_{n \in \mathbb{Z}} \alpha_{n} \mathcal{P}_{n} \mathcal{P}^{(k)}\right\|_{\infty} \leqslant \alpha_{k+1}
$$

The definition of the transformer $\Gamma_{k}$ implies the estimate

$$
\left\|\Gamma_{k}(X F)\right\|_{2}=\left\|\Gamma_{k}\left(X F \mathcal{P}^{(k)}\right)+\Gamma_{k}\left(\mathcal{P}^{(k)} X F \mathcal{P}_{(k)}\right)\right\|_{2} \leqslant \frac{\omega}{2 \pi} \alpha_{k+1}\|X\|_{2}+\left\|\Gamma_{k}\left(\mathcal{P}^{(k)} X F \mathcal{P}_{(k)}\right)\right\|_{2}
$$

We represent the operator $\Gamma_{k}\left(\mathcal{P}^{(k)} X F \mathcal{P}_{(k)}\right)$ as

$$
\Gamma_{k}\left(\mathcal{P}^{(k)} X F \mathcal{P}_{(k)}\right)=\Gamma_{k}\left(\mathcal{P}^{(k)} F X \mathcal{P}_{(k)}\right)+\Gamma_{k}\left(\mathcal{P}^{(k)}(X F-F X) \mathcal{P}_{(k)}\right) .
$$

At that, the latter operator has an operator matrix $\left(\mathcal{P}_{i} Z_{(k)} \mathcal{P}_{j}\right), i, j \in \mathbb{Z}, k \geqslant 0$, where

$$
Z_{(k)}=\Gamma_{k}\left(\mathcal{P}^{(k)}(X F-F X) \mathcal{P}_{(k)}\right)
$$


formed by the entries of form

$$
\mathcal{P}_{i} Z_{(k)} \mathcal{P}_{j}=\frac{f\left(\lambda_{i}\right)-f\left(\lambda_{j}\right)}{\lambda_{i}-\lambda_{j}} \mathcal{P}_{i} X \mathcal{P}_{j}=\frac{\omega}{2 \pi} \frac{\alpha_{i}-\alpha_{j}}{i-j} \mathcal{P}_{i} X \mathcal{P}_{j}
$$

where $|i| \geqslant k+1,|j| \leqslant k$, and $\mathcal{P}_{i} Z_{(k)} \mathcal{P}_{j}=0$ otherwise. Thus,

$$
\begin{aligned}
\left\|\Gamma_{k}\left(\mathcal{P}^{(k)} X F \mathcal{P}_{(k)}\right)\right\|_{2} & \leqslant \frac{\omega}{2 \pi} \alpha_{k+1}\|X\|_{2}+\frac{\omega}{2 \pi} \max _{\substack{|i| \geqslant k+1 \\
|j| \leqslant k}} \frac{\left|\alpha_{i}-\alpha_{j}\right|}{|i-j|}\|X\|_{2} \\
& =\frac{\omega}{2 \pi}\left(\alpha_{k+1}+\alpha_{k+1}^{\prime}\right)\|X\|_{2}=\widetilde{\alpha}_{k+1}\|X\|_{2} .
\end{aligned}
$$

In the same way the norm $\Gamma_{k}(F X), X \in \mathfrak{S}_{2}(\mathcal{H})$, is estimated. The proof is complete.

Lemma 8. A triple $\left(\mathcal{M}, J_{k}, \Gamma_{k}\right)$ is admissible for the unperturbed operator $L_{0}$ for each $k \geqslant 0$ and the constant $\gamma=\gamma_{k}$ in Definition 14 obeys the estimate

$$
\gamma_{k} \leqslant \widetilde{\alpha}_{k+1}, \quad k \in \mathbb{Z} .
$$

Proof. We have established above that the introduced space of admissible perturbations $\mathcal{M}$ is a Banach space. It follows from the embedding $\mathcal{M} \subset \mathfrak{S}_{2}(\mathcal{H})$ that $\mathcal{M}$ is embedded into $\mathfrak{L}_{L_{0}}(\mathcal{H})$ since each bounded operator is relatively bounded with respect to the unperturbed operator $L_{0}$. This is why Property 1 ) in Definition 14 holds true.

Properties 2) and 5) follow the construction of transformers $J_{k}, \Gamma_{k}, k \geqslant 0$, and Lemma 5 .

Properties 3) and 6) are checked in the same way as similar properties were established in Lemma 4.

We proceed to proof of property 4). Let $X=X_{l} F \in \mathcal{M}, Y=Y_{l} F \in \mathcal{M}$, where $X_{l}$, $Y_{l} \in \mathfrak{S}_{2}(\mathcal{H})$. Then $X \Gamma_{k} Y=Z_{l} F$, where $Z_{l}=X_{l} \Gamma_{k}\left(F Y_{l}\right)$. It follows from Lemma 5 that

$$
\left\|Z_{l}\right\|_{2} \leqslant \widetilde{\alpha}_{k+1}\left\|X_{l}\right\|_{2}\left\|Y_{l}\right\|_{2} \leqslant \widetilde{\alpha}_{k+1}\|X\|_{\mathcal{M}}\|Y\|_{\mathcal{M}} .
$$

Let $X=F X_{r}, Y=F Y_{r}, X_{r}, Y_{r} \in \mathfrak{S}_{2}(\mathcal{H})$. Then $X \Gamma_{k} Y=F Z_{r}$, where $Z_{r}=X_{r} \Gamma_{k}\left(F Y_{r}\right)$, and again it follows from Lemma 5 that

$$
\left\|Z_{r}\right\|_{2} \leqslant \widetilde{\alpha}_{k+1}\left\|X_{r}\right\|_{2}\left\|Y_{r}\right\|_{2} \leqslant \widetilde{\alpha}_{k+1}\|X\|_{\mathcal{M}}\|Y\|_{\mathcal{M}} .
$$

A similar estimate holds for the norm of the operator $\left(\Gamma_{k} X\right) Y$. The proof is complete.

Theorem 16. Let an integer $k \geqslant m$ be such that the condition

$$
4 \widetilde{\alpha}_{k+1}\|\widetilde{V}\|_{\mathcal{M}}<1
$$

is satisfied. Then the operator $L_{0}-\widetilde{V}$ is similar to the operator $L_{0}-J_{k} X_{*}=L_{0}-V_{0}$, where the operator $X_{*} \in \mathcal{M}$ is a solution to nonlinear operator equation (18) in Theorem 11 with transformers $J=J_{k}$ and $\Gamma=\Gamma_{k}$ defined by formulae (24), (25) and $B=\widetilde{V}$. Moreover, the operator $V_{0}$ is an orthogonal direct sum

$$
V_{0}=V_{0(k)} \oplus \bigoplus_{|i|>k} V_{0 i}
$$

with respect to the representation of the space $L_{2}$ of the form

$$
\mathcal{H}=\mathcal{H}_{(k)} \oplus \bigoplus_{|i|>k} \mathcal{H}_{i}
$$

where $\mathcal{H}_{(k)}=\operatorname{Im} P_{(k)}, \mathcal{H}_{i}=\operatorname{Im} P_{i},|i|>k$, and the projectors $P_{(k)}, P_{i},|i|>k$, are the spectral projectors of the unperturbed operator $L_{0}$ defined by formulae (13). The operator of transformation the operator $L_{0}-\widetilde{V}, \widetilde{V} \in \mathfrak{S}_{2}(\mathcal{H})$ into $L_{0}-V_{0}$ is the operator $I+\Gamma_{k} X$, where $X \in \mathcal{M} \subset \mathfrak{S}_{2}(\mathcal{H})$ and $\Gamma_{k} X \in \mathfrak{S}_{2}(\mathcal{H})$. 
Theorem 16 follows Theorems 11, 14, Lemmata 7, 8 and the property $\lim _{k \rightarrow \infty} \widetilde{\alpha}_{k}=0$, which ensures Condition (17) of Theorem 11 .

\section{Proof of Theorems 3, 4, 5}

Theorems 15 and 16 imply Theorem 3 given in Section 1 . We stress the operator $U$ in Theorem 3 is of the form

$$
U=U_{m k}=\left(I+\Gamma_{m} V\right)\left(I+\Gamma_{k} X_{*}\right)=I+W_{m k},
$$

where the operator $W_{m k}=\Gamma_{m} V+\Gamma_{k} X_{*}+\left(\Gamma_{m} V\right)\left(\Gamma_{k} X_{*}\right)$ belongs to $\mathfrak{S}_{2}(\mathcal{H})$.

We proceed to estimating the eigenvalues and the eigenprojectors. The next theorem follows Theorem 16 and Lemma 1.

Theorem 17. The spectrum of the operator $L$ coincides with the spectrum of the operator

$$
L_{0}-V_{0}=L_{0}-P_{(k)} X_{*} P_{(k)}-\bigoplus_{|j|>k} P_{j} X_{*} P_{j}
$$

Moreover, the identity holds:

$$
\sigma(L)=\sigma\left(L_{(k)}\right) \cup\left(\bigcup_{|j|>k} \sigma\left(L_{j}\right)\right)=\sigma\left(L_{(k)}\right) \cup\left\{i \frac{2 \pi j}{\omega}+x_{* j j},|j|>k\right\},
$$

where $L_{(k)}$ is the restriction of the operator $L_{0}-P_{(k)} X_{*} P_{(k)}$ on $\mathcal{H}_{(k)}=\operatorname{Im} P_{(k)}$ and $L_{j}$ is the restriction of the operator $L_{0}-P_{j} X_{*} P_{j}$ and $\operatorname{Im} P_{j},|j|>k$, while $x_{* j j},|j|>k$, are the diagonal entries of the matrix of the operator $X_{*}$.

Theorem 17 implies immediately Theorem 4. In Theorem 4, it is taken into consideration that for each bounded operator, its spectral radius does not exceeds the norm and the sequence $\left(x_{* j j}\right),|j|>k$, is square summable since $X_{*} \in \mathfrak{S}_{2}(\mathcal{H})$. All statements on the eigenvectors follow Lemma 1 and the representation of the invertible operator of transformation (28) as $U=I+\Gamma_{k m} X_{*}$, where $\Gamma_{k m} X_{*} \in \mathfrak{S}_{2}(\mathcal{H})$.

Proof of Theorem 5. The arguing is made under the assumptions of Theorem 3. We fix numbers $k, m \in \mathbb{Z}_{+}$such that the assumptions of Theorems 15, 16 hold. Let $\mathcal{P}_{n}=P\left(\left\{\lambda_{n}\right\}, L_{0}\right)$, $\lambda_{n}=\left\{\frac{i 2 \pi n}{\omega}\right\}, n \in \mathbb{Z}, \mathcal{P}_{(m)}=\sum_{|n| \leqslant m} \mathcal{P}_{n}, \widetilde{\mathcal{P}}_{(m)}=U_{k m} \mathcal{P}_{(m)} U_{k m}^{-1}, \widetilde{\mathcal{P}}_{n}=U_{k m} \mathcal{P}_{n} U_{k m}^{-1}$. According Lemma 1, the projectors $\widetilde{\mathcal{P}}_{(m)}$ and $\widetilde{\mathcal{P}}_{n}$ are the spectral projectors of the operator $L$. By the symbols $\mathcal{P}(\Omega)$ and $\widetilde{\mathcal{P}}(\Omega)$ we respectively denote the following projectors:

$$
\mathcal{P}(\Omega)=\sum_{n \in \Omega} \mathcal{P}_{n}, \quad \widetilde{\mathcal{P}}(\Omega)=\sum_{n \in \Omega} \widetilde{\mathcal{P}}_{n}=\sum_{n \in \Omega} U_{k m} \mathcal{P}_{n} U_{k m}^{-1}, \quad \Omega=\mathbb{Z} \backslash\{-m, \ldots, m\} .
$$

We note that $\mathcal{P}(\Omega)$ is a spectral projector constructed by the spectral set $\left\{\lambda_{n}, n \in \Omega\right\}$ of the operator $L_{0}$.

For each $X \in \mathfrak{S}_{2}(\mathcal{H})$ we define a quantity

$$
\alpha(\Omega, X)=\max _{n \in \Omega} \alpha_{n}(X), \quad \Omega \subset \mathbb{Z},
$$

where the sequence $\alpha_{n}, n \in \mathbb{Z}$, is defined by formula (27).

Let $X \in \mathcal{M}$, that is, $X=X_{l} F_{X}=F_{X} X_{r}$, where $X_{l}, X_{r} \in \mathfrak{S}_{2}(\mathcal{H})$. We estimate the norm

$$
\|\mathcal{P}(\Omega) X\|_{2}=\left\|\mathcal{P}(\Omega) F_{X} X_{r}\right\|_{2}=\left\|\left(\sum_{n \in \mathbb{Z}} \alpha_{n}(X) \mathcal{P}_{n}\right) X_{r}\right\|_{2} \leqslant \alpha(\Omega, X)\|X\|_{2}
$$

In the same way we obtain the estimate for the operator $X \mathcal{P}(\Omega)$. Therefore,

$$
\max \left\{\|\mathcal{P}(\Omega) X\|_{2},\|X \mathcal{P}(\Omega)\|_{2}\right\} \leqslant\|X\|_{\mathcal{M}} \alpha(\Omega, X) .
$$


We proceed to estimating the quantities $\left\|\mathcal{P}(\Omega) \Gamma_{m} X\right\|_{2},\left\|\Gamma_{m} X \mathcal{P}(\Omega)\right\|_{2}$ :

$$
\left\|\mathcal{P}(\Omega) \Gamma_{m} X\right\|_{2}^{2}=\sum_{\substack{i \in \Omega, j \in \mathbb{Z} \\ i \neq j}} \frac{\left\|\mathcal{P}_{i} \mathcal{P}(\Omega) X \mathcal{P}_{j}\right\|_{2}^{2}}{\left|\lambda_{i}-\lambda_{j}\right|^{2}} \leqslant\left(\frac{\omega}{2 \pi}\right)^{2} \sum_{i \in \Omega}\left\|\mathcal{P}_{i} X\right\|_{2}^{2} \leqslant\left(\frac{\omega}{2 \pi}\right)^{2} \alpha^{4}(\Omega, X)\|X\|_{2}^{2}
$$

Thus,

$$
\left\|\mathcal{P}(\Omega) \Gamma_{m} X\right\|_{2} \leqslant \frac{\omega}{2 \pi} \alpha^{2}(\Omega, X)\|X\|_{2} .
$$

In the same way and by the same quantity we estimate $\left\|\Gamma_{m} X \mathcal{P}(\Omega)\right\|_{2}$. We note that the definition of the sequence $\alpha$ and the constructing of the space of admissible perturbations $\mathcal{M}$ imply the identities $\|F \mathcal{P}(\Omega)\|=\|\mathcal{P}(\Omega) F\|=\alpha(\Omega, B)$. This is why $\alpha(\Omega, X) \leqslant\|X\|_{\mathcal{M}} \alpha(\Omega, B)$.

Also the definition of the sequence $\alpha_{n}, n \in \mathbb{Z}$, imply the following properties:

1) If $X=\sum_{l \geqslant 1} X_{l}$, where $X_{l} \in \mathfrak{S}_{2}(\mathcal{H})$, when this series converges absolutely and

$$
\|X\|_{2} \alpha_{n}(X) \leqslant \sum_{l \geqslant 1}\left\|X_{l}\right\|_{2} \alpha_{n}\left(X_{l}\right)
$$

2) If $X=X_{1} \cdots X_{l}, X_{j} \in \mathfrak{S}_{2}(\mathcal{H}), 1 \leqslant j \leqslant l$, then

$$
\|X\|_{2} \alpha_{n}(X) \leqslant\left(\alpha_{n}\left(X_{1}\right)+\cdots+\alpha_{n}\left(X_{l}\right)\right) \bigcap_{j=1}^{l}\left\|X_{j}\right\|_{2} .
$$

We proceed to estimating the norm of the difference $\widetilde{\mathcal{P}}(\Omega)-\mathcal{P}(\Omega)$, where $\Omega \subset \mathbb{Z} \backslash$ $\{-m, \ldots, m\}$. We recall that the operator $U$ in Theorem 3 making the similarity transformation can be represented as $U_{m k}=I+W_{m k}$, where $W_{m k}=\Gamma_{m} V+\Gamma_{k} X_{*}+\left(\Gamma_{m} V\right)\left(\Gamma_{k} X_{*}\right)$. Lemma 1 implies the identity

$$
\widetilde{\mathcal{P}}(\Omega)-\mathcal{P}(\Omega)=\left(W_{m k} \mathcal{P}(\Omega)-\mathcal{P}(\Omega) W_{m k}\right)\left(I+W_{m k}\right)^{-1} .
$$

Then

$$
\begin{aligned}
\left\|\mathcal{P}(\Omega) W_{m k}\right\|_{2} & \leqslant\left\|\mathcal{P}(\Omega)\left(\Gamma_{m} V\right)\right\|_{2}+\left\|\mathcal{P}(\Omega)\left(\Gamma_{k} X_{*}\right)\right\|_{2}+\left\|\mathcal{P}(\Omega)\left(\Gamma_{m} V\right)\left(\Gamma_{k} X_{*}\right)\right\|_{2} \\
& \leqslant\|\mathcal{P}(\Omega)(\Gamma V)\|_{2}+\left\|\mathcal{P}(\Omega)\left(\Gamma X_{*}\right)\right\|_{2}+\left\|\mathcal{P}(\Omega)(\Gamma V)\left(\Gamma X_{*}\right)\right\|_{2} \\
& \leqslant C_{1}\left(\alpha(\Omega, \Gamma V)+\alpha^{2}\left(\Omega, X_{*}\right)\right) \leqslant C_{2}\left(\alpha(\Omega, \Gamma V)+\alpha^{2}(\Omega, V)\right) .
\end{aligned}
$$

We note that the constants $C_{1}$ and $C_{2}$ are independent of $\Omega$. By a similar quantity we can estimate the norm $\left\|W_{m k} \mathcal{P}(\Omega)\right\|_{2}$.

The operator $\left(I+W_{m k}\right)^{-1}$ can be represented as $\left(I+W_{m k}\right)^{-1}=I+\sum_{j=1}^{\infty}(-1)^{j} W_{m k}^{j}$, this is why

As a result we obtain

$$
\left\|\left(I+W_{m k}\right)^{-1}-I\right\|_{2} \leqslant \frac{\left\|W_{m k}\right\|_{2}}{1-\left\|W_{m k}\right\|_{2}}
$$

$$
\|\widetilde{\mathcal{P}}(\Omega)-\mathcal{P}(\Omega)\|_{2} \leqslant C_{3}\left(\alpha(\Omega, \Gamma V)+\alpha^{2}(\Omega, V)\right),
$$

where the constant $C_{3}$ is independent of $\Omega$.

We observe that there two partitions of the unity in the space $\mathcal{H}$

$$
I=\sum_{|i|>k} \mathcal{P}_{i}+\mathcal{P}_{(k)}, \quad I=\sum_{|i|>k} \widetilde{\mathcal{P}}_{i}+\widetilde{\mathcal{P}}_{(k)} .
$$

The statement of Theorem 5 follows immediately estimate 29 if as $\Omega$ we consider the set $\Omega=\{n \in \mathbb{Z},|n|>N\}$, where $N$ is a sufficiently large natural number, and Property 2 ) of the sequence $\left\{\alpha_{n}\right\}$. 
Corollary 1. The operator $L$ is a spectral operator of scalar type (see [45]).

\section{Proof of Theorems 1, 6, 8}

We employ Theorem 3 and its more detailed version, Theorem 16, in order to construct a group of the operators $T: \mathbb{R} \rightarrow$ End $L_{2}$, whose generator is the studied operator with the involution $L: W_{2}^{1} \subset L_{2} \rightarrow L_{2}$. It follows from Theorem 16, Theorem 3, 12 and Lemma 2 that the group of the operators $T(t), t \in \mathbb{R}$, is an $U=U_{m k}$-orthogonal direct sum

$$
T(t)=U\left(\bigoplus_{j=-\infty}^{-k-1} e^{\left(\frac{i 2 \pi j}{\omega}+x_{* j j}\right) t} \oplus e^{X_{*(m)} t} \bigoplus_{j=k+1}^{\infty} e^{\left(\frac{i 2 \pi j}{\omega}+x_{* j j}\right) t}\right) U^{-1}, \quad t \in \mathbb{R},
$$

with respect to the $U$-orthogonal expansion

$$
L_{2}=\mathcal{H}=\bigoplus_{j=-\infty}^{-k-1} U \mathcal{H}_{j} \oplus U \mathcal{H}_{(k)} \oplus\left(\bigoplus_{j=k+1}^{\infty} U \mathcal{H}_{j}\right)
$$

We note that the numbers $m, k \in \mathbb{Z}_{+}$, are defined in Theorems 15 and 16 , respectively. At that, the operator $U_{m k}$ can be represented as $(28)$, that is, $U_{m k}=I+W_{m k}$, where $W_{m k} \in \mathfrak{S}_{2}(\mathcal{H})$.

Thus, we have constructed a group $T: \mathbb{R} \rightarrow$ End $L_{2}$, whose generator is the operator $L=$ $L_{0}-V$. The statement of Theorem 1 on representation of classical and mild solutions to problem (1) follows the general theory of semi-groups of the operators (see [29], [30], [45]). The proved representation (30) implies the statement of Theorem 6, where $b_{j}=x_{j j},|j| \geqslant m+1$, $B_{(k)}=X_{*}$. We note that Theorems 3,16 imply the property $\sum_{|j| \geqslant m+1}\left|b_{j}\right|^{2}<\infty$.

In order to prove Theorem 8, we employ representation (30) of the group $T$ and the Parseval identity. The made estimates are in fact given in the formulation of Theorem 8 ,

In conclusion we mention that the results of the present work were partially announced in [46] and [47]. The study in [48] follows the lines of the present work.

\section{BIBLIOGRAPHY}

1. I.C. Gohberg, M.G. Krein. Introduction to the theory of linear nonselfadjoint operators in Hilbert space, Nauka, Moscow (1965). [Am. Math. Soc., Providence, RI (1969).]

2. M.Sh. Burlutskaya, A.P. Khromov. Fourier method in an initial-boundary value problem for a firstorder partial differential equation with involution // Zhurn. Vychisl. Matem. Matem. Fiz. 51:12, 2233-2246 (2011). [Comp. Math. Math. Phys. 51:12, 2102-2114 (2011).]

3. M.Sh. Burlutskaya, A.P. Khromov. Initial-boundary value problems for first-order hyperbolic equations with involution // Dokl. RAN. 441:2, 151-154 (2011). [Dokl. Math. 84:3, 783-786 (2011).]

4. M.Sh. Burlutskaya. Mixed problem for a first-order partial differential equation with involution and periodic boundary conditions // Zhurn. Vychisl. Matem. Matem. Fiz. 54:1, 3-12 (2014). [Comp. Math. Math. Phys. 54:1, 1-10 (2014).]

5. M.Sh. Burlutskaya, A.P. Khromov. Mixed problem for simplest hyperbolic first order equations with involution // Izv. Saratov. Univ. Nov. Ser. Matem. Mekh. Inform. 14:1, 10-20 (2014). (in Russian).

6. L.V. Kritskov, A.M. Sarsenbi. Spectral properties of a nonlocal problem for a second-order differential equation with an involution // Differen. Uravn. 51:7, 990-996 (2015). [Diff. Equat. 51:7, 984-990 (2015).]

7. L.V. Kritskov, A.M. Sarsenbi. Basicity in $L_{p}$ of root functions for differential equations with involution // Electr. J. Differ. Equat. 278:278, 1-9 (2015).

8. L.V. Kritskov, A.M. Sarsenbi. Riesz basis property of system of root functions of second-order differential operator with involution // Differen. Uravn. 53:1, 35-48 (2017). [Diff. Equat. 53:1, 33-46 (2017).] 
9. J. Wiener, A.R. Aftabizadeh. Boundary value problems for differential equations with reflection of the argument // Intern. J. Math. Math. Sci. 8:1, 151-163 (1985).

10. D. Piao. Periodic and almost periodic solutions for differential equations with reflection of the argument // Nonl. Anal. 57:4, 633-637 (2004).

11. A. Cabada, F.A.F. Tojo. Existence results for a linear equations with reflection, non-constant coefficient and periodic boundary conditions // J. Math. Anal. Appl. 412:1, 529-546 (2014).

12. A. Cabada, F.A.F. Tojo. Solutions and Green's function of the first order linear equation with reflection and initial conditions // Boundary Value Probl. 99, 1-16 (2014).

13. W. Watkins. Asymptotic properties of differential equations with involutions // Int. J. Pure. Appl. Math. 44:4, 485-492 (2008).

14. A.A. Kopzhassarova, A.L. Lukashov, A.M. Sarsenbi. Spectral properties of non-self-adjoint perturbations for a spectral problem with involution // Abstr. Appl. Anal. 2012, id 590781 (2012).

15. A.A. Kopzhassarova, A.M. Sarsenb. Basis properties of eigenfunctions of second-order differential operators with involution // Abstr. Appl. Anal. 2012, id 576843 (2012).

16. M.A. Sadybekov, A.M. Sarsenbi. Criterion for the basis property of the eigenfunction system of a multiple differentiation operator with an involution // Differ. Uravn. 48:8, 1126-1132 (2012). [Diff. Equat. 48:8, 1112-1118 (2012).]

17. A.G. Baskakov, I.A. Krishtal, E.Yu. Romanova. Spectral analysis of a differential operator with an involution // J. Evolut. Equat. 17:2, 669-684. (2017).

18. A.G. Baskakov. Methods of abstract harmonic analysis in the perturbation of linear operators // Sibir. Matem. Zhurn. 24:1, 21-39 (1983). [Siber. Math. J. 24:1, 17-32 (1983).]

19. A.G. Baskakov. A theorem on splitting an operator, and some related questions in the analytic theory of perturbations // Izv. AN SSSR. Ser. Matem. 50:3, 435-457 (1986). [Math. USSR. Izv. 28:3, 421-444 (1987).]

20. A.G. Baskakov. Spectral analysis of perturbed nonquasianalytic and spectral operators // Izv. RAN. Ser. Matem. 58:4, 3-32 (1994). [Izv. Math. 45:1, 1-31 (1995).]

21. A.G. Baskakov, A.V. Derbushev, A.O. Shcherbakov. The method of similar operators in the spectral analysis of non-self-adjoint Dirac operators with non-smooth potentials // Izv. RAN. Ser. Matem. 75:3, 3-28 (2011). [Izv. Math. 75:3, 445-469 (2011).]

22. A.G. Baskakov, D.M. Polyakov. The method of similar operators in the spectral analysis of the Hill operator with nonsmooth potential // Matem. Sborn. 208:1, 3-47 (2017). [Sb. Math. 208:1, 1-43 (2017).]

23. R.E. Kalman, R.S. Bucy. New results in linear filtering and prediction theory // Trans. ASMI. Ser. D.J. Basic Eng. 83:1, 95-108 (1961).

24. D. Przeworska-Rolewicz. Equations with transformed argument: Algebraic approach. Polish Sci. Publ., Warsaw (1973).

25. V.A. Pliss. Nonlocal problems of the theory of oscillations. Nauka, Moscow (1964). [Academic Press, New York (1966).]

26. J. Wu. Theory and applications of partial functional differential equations. Springer, New York (1996).

27. W. Arendt, C.J.K. Betty, M. Hieber, F. Neubrander. Vector-valued Laplace transforms and Cauchy problems. Birkhäuser, Basel (2011).

28. S.G. Krein. Linear equations in Banach spaces. Nauka, Moscow (1967). [Birkhäuser, Boston (1982).]

29. E. Hille, R.S. Phillips. Functional analysis and semi-groups. Am. Math. Soc., Providence, RI (1962).

30. K.-J. Engel, R. Nagel One-parameter semigroups for linear evolution equation. Springer, New York (2000).

31. A.G. Baskakov, V.B. Didenko. Spectral analysis of differential operators with unbounded periodic coefficients // Differ. Uravn. 51:3, 323-341 (2015). [Differ. Equat. 51:3, 325-341 (2015).]

32. A.G. Baskakov, L. Yu. Kabantsova, I.D. Kostrub, T.I. Smagina. Linear differential operators and operator matrices of the second order // Differ. Uravn. 53:1, 10-19 (2017). [Diff. Equat. 53:1, 8-17 (2017).] 
33. A.G. Baskakov. Spectral criteria for almost periodicity of solutions of functional equations // Matem. Zamet. 24:2, 195-206 (1978). [Math. Notes. 24:2, 606-612 (1978).]

34. B.M. Levitan, V.V. Zhikov. Almost periodic functions and differential equations. Izdat. MGU, Moscow. [Cambridge Univ. Press, Cambridge (1982)]

35. A.G. Baskakov. Analysis of linear differential equations by methods of the spectral theory of difference operators and linear relations // Uspekhi Matem. Nauk. 68:1(409), 77-128 (2013). [Russ. Math. Surv. 68:1, 69-116 (2013).]

36. A.G. Baskakov. Harmonic and spectral analysis of power bounded operators and bounded semigroups of operators on Banach spaces // Matem. Zamet. 98:2, 174-190 (2015). [Math. Notes. 98:2, 164-178 (2015).]

37. A.G. Baskakov. Estimates for the Green's function and parameters of exponential dichotomy of a hyperbolic operator semigroup and linear relations // Matem. Sborn. 206:8, 23-62 (2015). [Sb. Math. 206:8, 1049-1086 (2015).]

38. D.M. Polyakov. Spectral analysis of a fourth order differential operator with periodic and antiperiodic boundary conditions // Alg. Anal. 27:5, 117-152 (2015). [St.-Petersburg Math. J. 27:5, 789-811 (2016).]

39. N.B. Uskova. On spectral properties of Sturm-Liouville operator with matrix potential // Ufimskij Matem. Zhurn. 7:3, 88-99 (2015). [Ufa Math. J. 7:3, 84-94 (2015).]

40. N.B. Uskova. On the spectral properties of a second-order differential operator with a matrix potential // Differ. Uravn. 52:5, 579-588 (2016). [Diff. Equat. 52:5, 557-567 (2016).]

41. G.V. Garkavenko, N.B. Uskova. Spectral analysis of a class of difference operators with growing potential // Izv. Saratov. Univ. Nov. Ser. Matem. Mekh. Inform. 16:4, 395-402 (2016). (in Russian).

42. G.V. Garkavenko, N.B. Uskova. Method of similar operators in research of spectral properties of difference operators with growthing potential // Sib. Elektron. Matem. Izv. 14, 673-689 (2017).

43. A.G. Baskakov. Estimates for the entries of inverse matrices and the spectral analysis of linear operators // Izv. RAN. Ser. Matem. 61:6, 3-26 (1997). [Izv. Math. 61:6, 1113-1135 (1997).]

44. A.G. Baskakov, I.A. Krishtal. Memory estimation of inverse operators // J. Funct. Anal. 267:8, 2551-2605 (2014).

45. N. Dunford, J.T. Schwartz. Linear operators. Part I: General theory.. Interscience Publ., New York (1958).

46. A.G. Baskakov, N.B. Uskova. A generalized Fourier method for the system of first-order differential equations with an involution and a group of operators // Differ. Uravn. 54:2, 276-280 (2018). [Diff. Equat. 54:2, 277-281 (2018).]

47. A.G. Baskakov, N.B. Uskova. Spectral analysis of differential operator with an involution and a group of operators // Differ. Uravn. 54:9, 1287-1291 (2018). [Diff. Equat. 54:9, (2018), to appear.]

48. A.G. Baskakov, I.A. Krishtal, N.B. Uskova. Linear differential operator with an involution as a generator of an operator group // Operators and Matrices. 12:3, 723-756 (2018).

Anatoly Grigorievich Baskakov,

Voronezh State University,

Universitetskaya sq. 1,

394018, Voronezh, Russia

E-mail: anatbaskakov@yandex.ru

Natalia Borisovna Uskova,

Voronezh State Technical University,

Moskovsky av. 14,

394016, Voronezh, Russia

E-mail: nat-uskova@mail.ru 